\title{
Looking at Islamic Patterns I: The Perception of Order
}

\author{
Peter R. Cromwell
}

28 July 2021

http://girih.wordpress.com

\begin{abstract}
Islamic geometric patterns are complex arrangements of interlocking stars and polygons. They are cultural artefacts rather than stimuli specifically devised for scientific experiments, but they provide a good corpus of test cases to study the human visual system: they have few confounding variables, and contain examples that display convincing combinations of incompatible shapes in configurations that are geometrically impossible. The literature on vision leaves some of their properties unexplained.

We define regularities as features of visual data that are processed fluently, and hence depend on properties of the stimulus and the viewer. Useful information is found in non-accidental relationships, and these seem to correspond to regularities for low-level structure that is expected to be seen in the environment. We find that qualitative properties are more salient than metric properties: misalignment between neighbouring stars is more noticeable than imperfect symmetry of individual stars, for example. We develop a model of local symmetry centres, which predicts that stars with odd or even numbers of spikes have different visual properties, and this influences where and how stars should be positioned in a pattern for best effect.
\end{abstract}




\section{Introduction}

Why do we look at geometric patterns? What attracts us, gives us pleasure, and retains our interest? These questions concern our interaction with and response to works of decorative art, and the answers depend on properties of both the viewer and the pattern. Moreover, the visual system is adapted for survival, so our questions also engage the broader ecosystem in which it developed. As Wood points out [74, 75], part of the appeal of patterns derives from the sense of processing fluency they afford. For example, patterns (by definition) contain repetition, and the stars in Islamic patterns provide recognisable landmarks that make the repetition easy to find. Predictable structure is easy to process, and successful processing in itself is rewarding for the viewer. We shall explore in greater detail what geometric patterns tell us about the working of the human visual system, and which properties of Islamic geometric patterns make some more successful than others.

Islamic geometric ornament provides a rich source of visual data. A large corpus containing thousands of patterns covers the area from India and Central Asia through Persia and North Africa to Spain, and the evolution of these patterns from the twelfth to the eighteenth centuries displays constant innovation. Yet, despite the variety of media and approaches, this large data set is coherent - the characteristic interlocking stars and polygons are always immediately recognisable.

This is the first of two papers, and the division between them can be thought of in various ways. Depending on your background you might think of the two stages as early and late vision, perception and cognition, or signal processing and information processing. In the sense that the first stage is data driven and depends on the properties of the pattern being observed while the second stage depends to some degree on the knowledge and experience of the viewer, you can also think of the two stages as being objective and subjective. The emotional responses generated by the two stages are often referred to as pleasing and interesting.

Islamic geometric patterns differ from the typical stimuli used in psychology experiments in their level of complexity. A single pattern can contain different kinds of structure, longand short-range structure, even hierarchical structure. Figure 1 shows an example designed by the author in a style popular in Cairo during the Mamluk period. It is a mesh of interlinked convex polygons, most of which are kites. The design has various high-level structures: the large voids are star-shaped with 13 or 17 points, and (imaginary) lines connecting neighbouring star centres form axes of local or global symmetry. If you try to copy the pattern, you will discover that what you perceive is not what is drawn. If you are familiar with the mathematics of repeating patterns then you will know that motifs with 13-fold and 17-fold symmetry do not fit together as shown. Illicit patterns of this type were produced by medieval artists, pushing against the constraints imposed by geometry. In our study of the visual system, such patterns enable us to explore the relative importance of different properties when they cannot be achieved simultaneously.

This paper does not assume the reader has a background in either Islamic patterns or visual perception. The case study in $\S 2$ introduces basic terminology for patterns and deconstructs an example. Here we are not trying to recover detailed geometric constructions for laying out a pattern; we are interested in working out which choices were made by the artist and what effect they have on the viewer.

The scientific exploration of how we convert the changing pattern of light falling onto the 


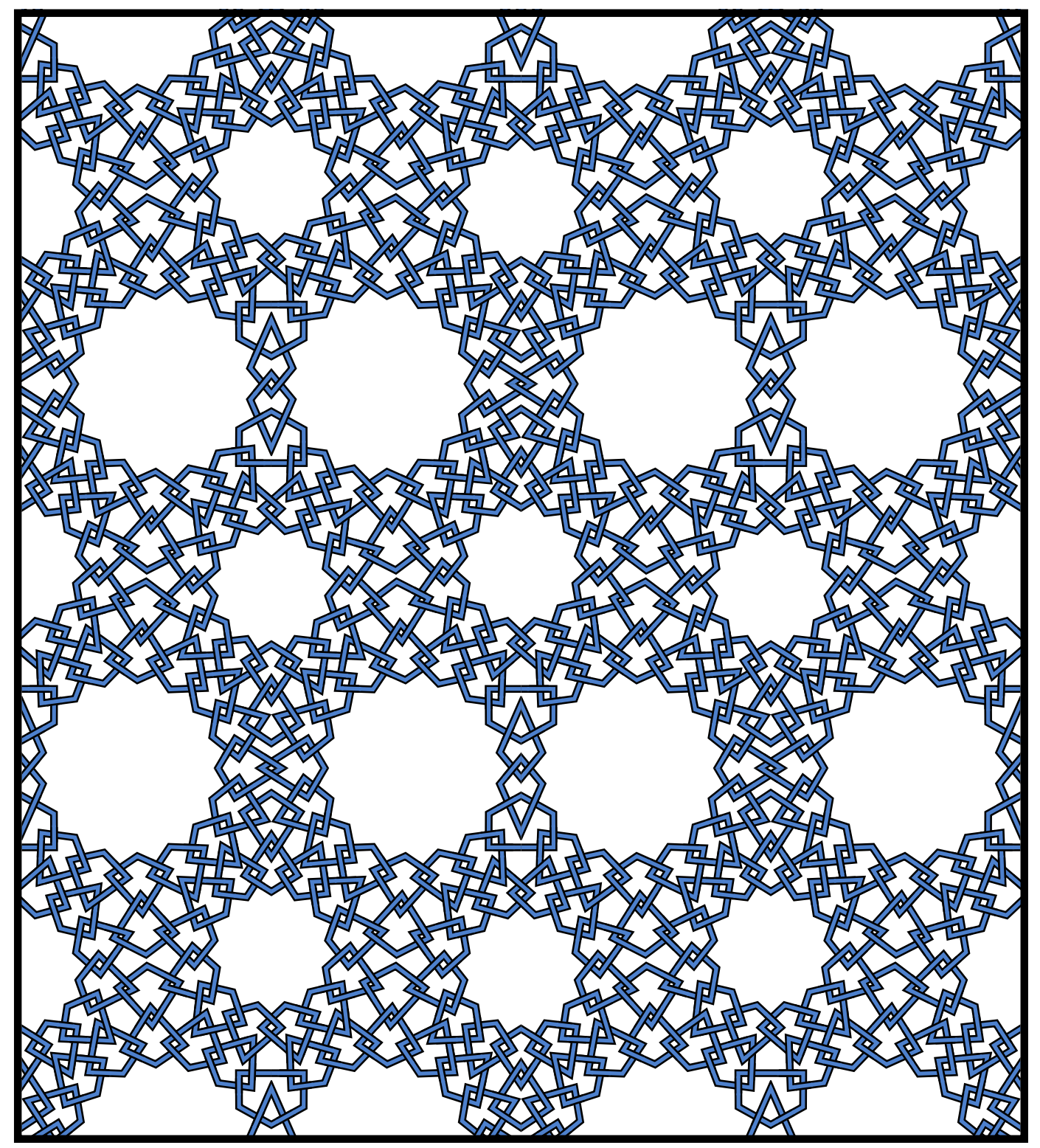

Figure 1: Design by the author. The style used here, characterised by interlinked kites, was popular in the Mamluk period. The stars have 13 or 17 points. (c) P. R. Cromwell. 
retinae in our eyes into knowledge of our environment has generated an extensive literature. The fundamentals of visual perception are discussed in $[9,18,25]$. Several books and articles deal specifically with the psychology of art $[3,10,23,49,50,52,55,59,68,76]$ or ornamental patterns $[24,70]$. In $\S 3$ we review elements of psychophysics and perceptual organisation pertinent to geometric patterns. It will become apparent that there are close parallels between pattern analysis and visual perception, in particular, the importance of non-accidental properties in both processes.

The remainder of the paper explores the perception of order in geometric patterns, particularly in Islamic star patterns. We identify different sources of order and try to discriminate between the routine structure that arises from a production mechanism, and more deliberate acts on the part of the artist. There are sources of ambiguity, and strong features may be emergent rather than designed. We review a technique used in pattern analysis for finding structure, and expose its benefits and limitations. In trying to understand what makes a good star, we present a new perceptual model of regularity for motifs with a centre of symmetry, and make predictions about relative goodness. Finally, we bring the threads together to explore what makes a good pattern. We show what happens when stars are assembled, prioritising structural features in different ways, and explain the conflicts and resolutions used to create Figure 1.

All the examples except for Figure 1 are from historical sources in Persia or Central Asia.

\section{Islamic geometric patterns}

At first glance, an Islamic star pattern is just a large matrix of interlocking polygonal shapes. Yet it is not disorganised, and neither the shapes nor their arrangement is random. There is a lot of local and global structure to be discovered, and the pattern presents puzzles for the viewer: what are the raw ingredients and how do they fit together?, how was something so intricate designed and constructed? Islamic patterns also pose problems for the historian as there are no surviving documents that explain the grammar of Islamic style - we have to recover the traditional rules and design principles through observation and analysis.

As an introduction to the properties of Islamic patterns we will work through a case study, making sense of the visual data. The example chosen is not typical and exhibits a variety of unusual features. We shall, however, be able to explain the presence and form of the anomalies and assign them to different causes, something that increases confidence in the analysis and the proposed reconstruction. (It does not matter if you lose track of the details - the key thing is that useful information is a consequence of situations that are unlikely to arise by chance.)

Figure 2 shows a wall panel from the south iwan in the Friday Mosque in Isfahan, Iran. The panel is partitioned into polygonal cells, each of which is filled with a decorative design made by arranging small fragments cut from glazed ceramic tiles. The tiles are monochromatic, so each colour can be fired independently at its optimum temperature, which gives the panel a vibrant finish. The four octagonal cells contain a hub-and-spokes design with Kufic calligraphy in brown on a black ground. The other cells display floral arabesques on a black, dark blue or light blue ground. The tiles contrast with the beige, matt uniformity of the unglazed strips outlining the polygons.

In Figure 3(a), the colours and decoration have been discarded to highlight the polygonal 


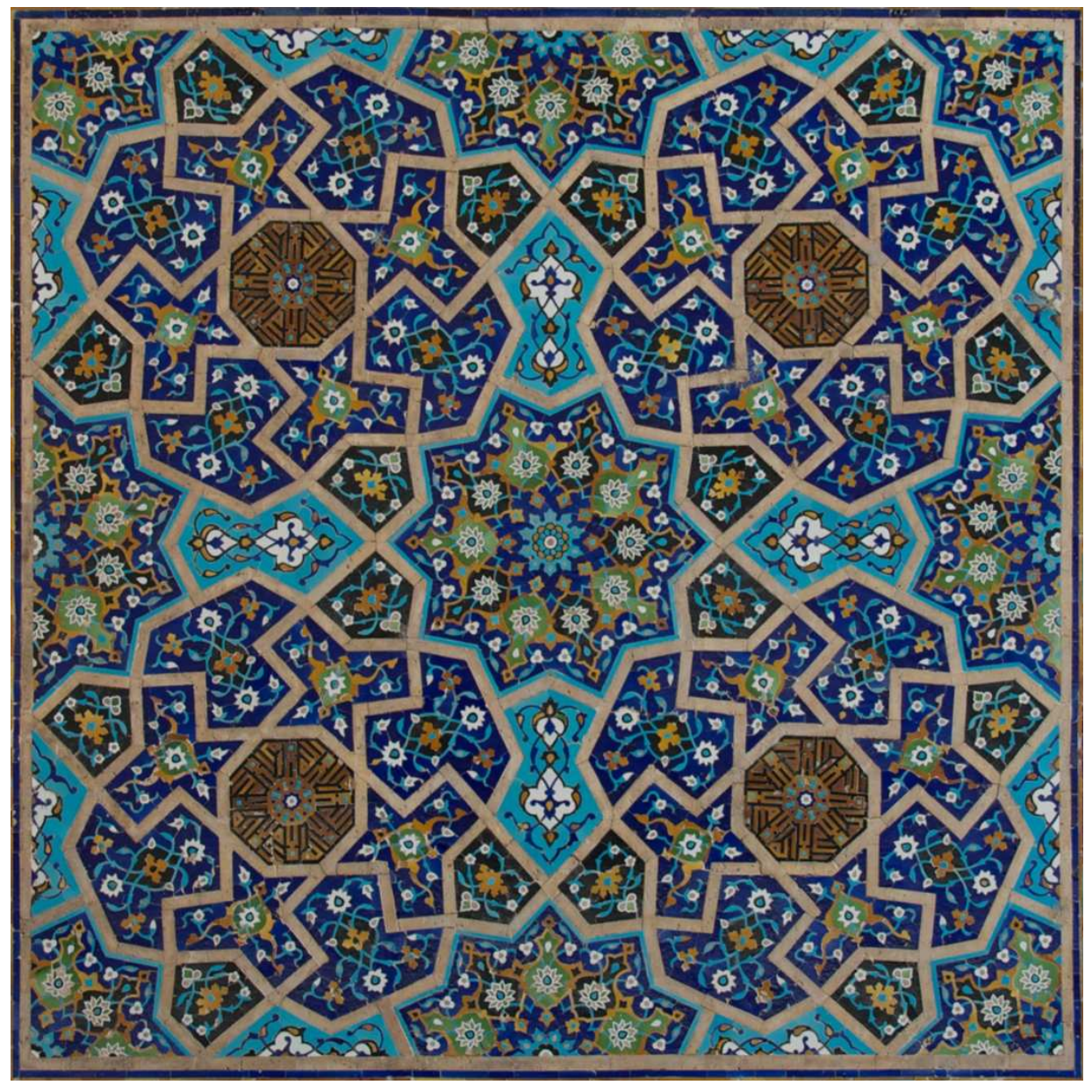

Figure 2: A tiled panel from the south iwan (1475) in the Friday Mosque, Isfahan, Iran. 


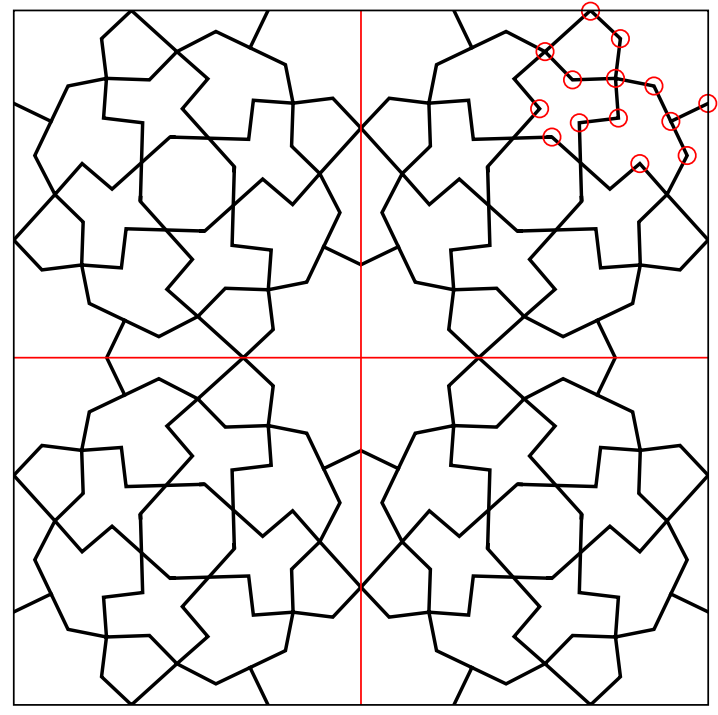

(a)

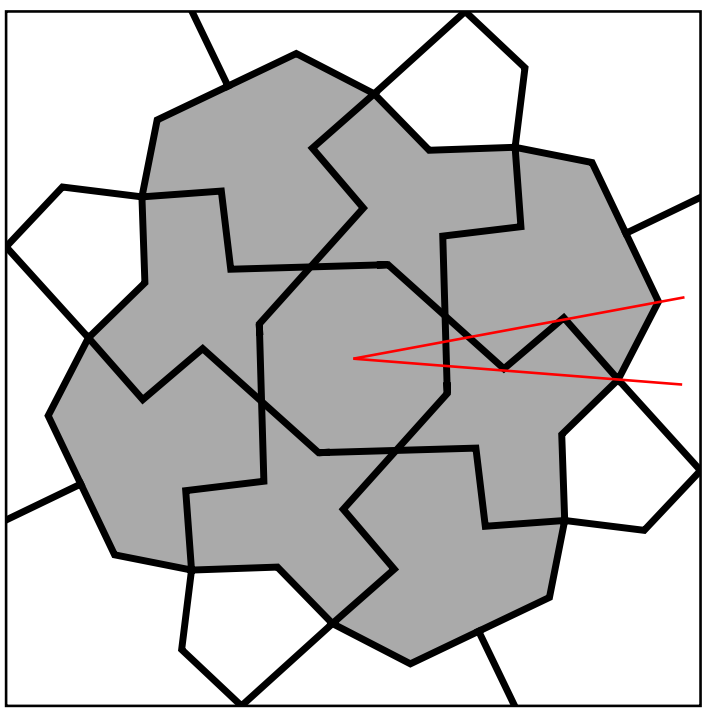

(b)

Figure 3: First steps in the analysis of Figure 2.

cell structure of the design. The panel exhibits a quartering that is quite common in square and rectangular panels: it has both vertical and horizontal reflection symmetries (the mirror lines are shown in red). The top-right quadrant is reproduced at a larger scale in Figure 3(b); it clearly has 4-fold rotational symmetry. This hierarchical decomposition using symmetry transformations means that the whole pattern can be specified by giving a small amount of information. In fact, the locations of the fourteen points circled in red, the connections between them, and the repetition rules are sufficient.

Let us focus on the structure of Figure 3(b). The points where line segments meet are called vertices. A vertex that is the end-point of $n$ line segments is called $n$-valent. The 4 -valent vertices formed at the intersection of two straight lines are called transverse. Most vertices in most Islamic patterns are transverse crossings. In this pattern, some of the vertices are 3-valent and have the form of T-junctions. Furthermore, some of the 4 -valent vertices are not transverse as lines deviate from a straight path as you travel through the vertex.

One aim in analysing a pattern is to discriminate features that are the results of decisions made by the artist from those that are imposed by laws of geometry or due to other causes. Close inspection of Figure 3(b) shows that the corners of some the cells lie on radial lines (shown in red), something which seems unlikely to have happened by chance. Some of the angles look close to right angles but are slightly off, the regular octagonal cells are set at a strange angle with respect to the boundary of the panel, and some of what appear to be 4-valent vertices are seen to be staggered junctions formed from two 3 -valent vertices close together. The misalignment at the staggered junctions displays random variations across the panel, so is probably a manufacturing defect. However, the deviation at 4 -valent vertices and the other unusual features are consistent throughout the panel and they require explanation.

Figure 4(a) shows the construction of a simple zig-zag that someone may doodle in an idle moment. The red lines originate at the point $O$ in the centre of the octagon; the two 


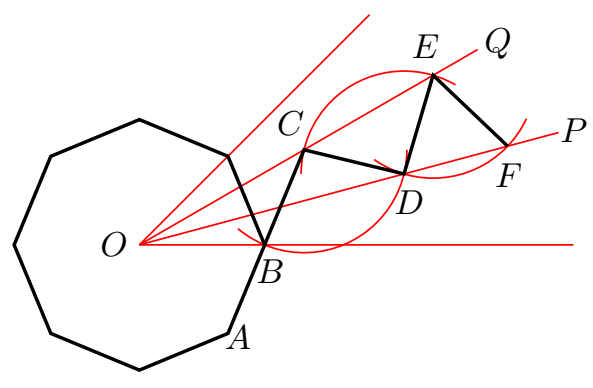

(a)

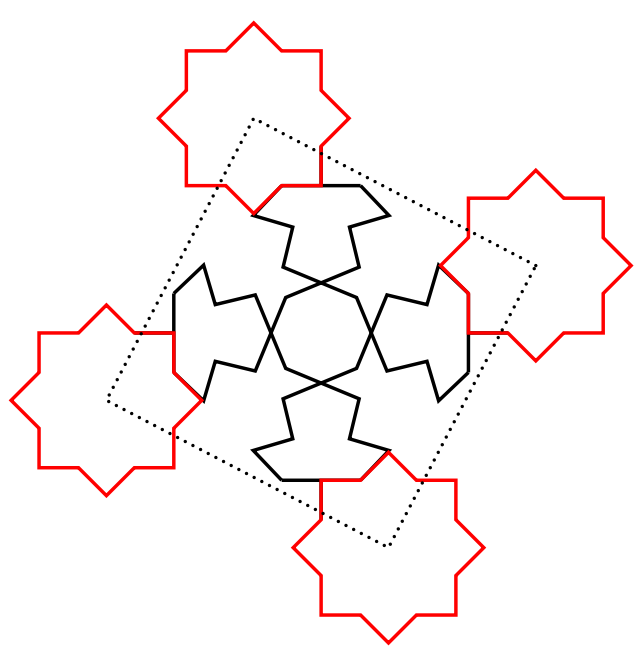

(c)

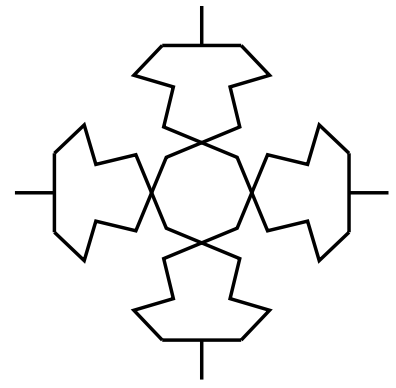

(b)

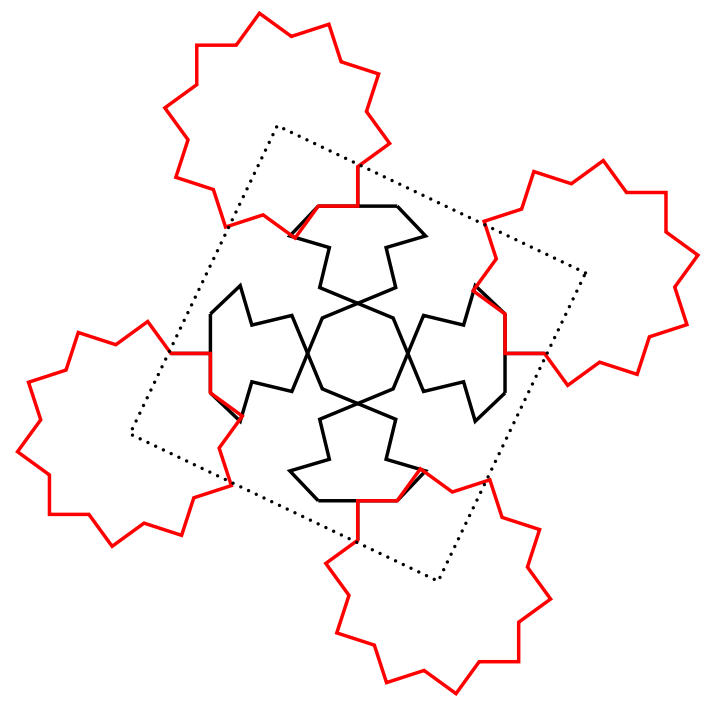

(d)

Figure 4: A possible starting point for the composition of Figure 2. 
outer lines pass through adjacent vertices of the octagon, and the other two, $O P$ and $O Q$, divide the $45^{\circ}$ sector into three equal parts. The side $A B$ of the octagon is extended until it meets line $O Q$ to produce point $C$. Place compasses centred at $C$ and scribe an arc through $B$ until it crosses the line $O P$, producing point $D$. Place compasses centred at $D$ and scribe an arc through $C$ until it crosses the line $O Q$, producing point $E$. Repeat again to obtain $F$.

This zig-zag line can be repeated to generate the motif in Figure 4(b), which clearly forms a good starting point for the composition. By construction, the edges $B C, C D, D E$ and $E F$ have the same length, and are slightly longer than $A B$; the angle $\measuredangle C D E \approx 92.8^{\circ}$ is slightly larger than a right angle. So, for the cell corners that lie on radial lines, simply marking off lines of equal length creates shapes and angles that match the archaeological data closely.

The design is developed by combining this 4-fold motif with stars. Figure 4(c) and (d) shows the effects of adding 8-pointed and 10-pointed stars. There are two aspects to consider: how the stars relate to the motif, and how they relate to each other. The angles in the 8-pointed stars match the motif better, but the alignment of the 10-pointed stars with each other is better. The artist completed the design with 10-pointed stars, and used the square determined by their centres to define the extent of the template. The fact that the octagons are not aligned with the frame is an incidental consequence of this decision. The design is completed by partitioning the residual space to produce cells with bilateral symmetry.

This generative process also explains some of the anomalies in the pattern, mostly as a consequence of the fact that the 10-pointed stars and the central motif do not fit together. Look at the shaded area in Figure 3(b): the 3-valent vertices and the non-transverse 4-valent vertices all occur along its boundary contour where the two incompatible motifs collide. Notice that the shaded area is also considered as a single unit in Figure 2 where (except for the central octagon) it has a uniform dark blue ground.

We have seen that the structure in this panel occurs in different forms, at a range of scales, and for various reasons. We identified structure by looking for geometric features that are unlikely to arise by chance: three or more points lying on a straight line, four or more points on a circle, equal spacing, consistent configuration at multiple locations. This led to the abstraction of motifs and the relationships between them. The rest of the paper explores which properties are important for visual impact, and which are under the control of the artist.

\section{Perceptual organisation and psychophysics}

When you look at an unfamiliar geometric pattern, you do not see the raw ingredients, the lines, corners and crossings. As your eye scans the pattern, areas of interest and complete motifs pop out spontaneously. This subconscious process of giving structure to sensory data is called perceptual organisation. It is one of the early stages of our visual system, has been intensively studied, and has a large literature. This section introduces the ideas and terminology needed for the following discussion. See [46, 54, 61, 73] for further information on perceptual organisation in general, and $[5,58]$ on the perception of symmetry!

\footnotetext{
${ }^{1}$ Note that in most papers on perception, the term 'symmetry' has a very narrow focus and means bilateral symmetry, usually with a vertical mirror. The papers cited here cover symmetry in the broader
} 
Perceptual organisation covers a range of problems.

- The simplest processes operate over a small area so they have short-range effects. They include local filters for locating boundaries at discontinuities between homogeneous regions, interpolating smoothly between line segments to bridge small gaps, and clustering — grouping items purely by proximity.

- Long-range processes can group items with shared attributes. Those based on primitive features include grouping items that lie inside a closed contour, grouping items by similarity of colour or orientation, and grouping segments that lie on a smooth line (good continuation).

- Grouping can also be performed on higher-order attributes like shape, or predicates combining several features. The grouped entity does not need to have a name or meaning, but familiarity with it or recent exposure improves the response time. The process can also be primed for selective attention: for example 'focus on red 5-pointed stars'.

- Besides placing related items in a group, long-range processing can also extract relationships between items. For symmetry transformations the relationships are quite strict and detailed but, in other cases, a more general schema can be sufficient for example, a particular configuration of blobs inside a closed contour can create a face. Configuration is not only concerned with position and arrangement, but also with 'part of' relationships and structural hierarchies.

- While configurations and hierarchies may correspond to analogous structures in the viewed environment, we can also find purely abstract features of the data that are defined only in terms of relationships. Even when there are no marks in the image to indicate the presence or location of symmetry elements such as mirror lines and rotation centres, we can still rely on internal representations of them and imagine them.

We can abstract the rules of repetition from a single pattern and find flaws where they are violated [70], yet it seems that we cannot match configurations of symmetry elements between patterns. Non-specialists cannot discriminate between four basic symmetry types: a periodic pattern of translations only $(p 1)$, and three types with the addition of a single kind of symmetry operation $(p 2, p m, p g)$ [37].

The processes in this summary have become more abstract down the list. Experiments show that some of simpler processes seem to be hard-wired and are either innate or trained very early in life by exposure to visual data. They are automatic and produce perceptions that do not always match the structure in the data, imposing structure on noise, and creating virtual objects. We cannot disable these responses, even when we understand how they work. For example, the star pattern in Figure 5 is composed of straight line segments, yet we see illusory curves flowing across the panel where the segments have been linked and interpolated to produce emergent objects [31].

Our visual system evolved over thousands of years of prehistory because it is useful for survival: flickering patterns of light energy arriving at receptors in our eyes provide data

sense that includes rotation centres and multiple mirrors. 


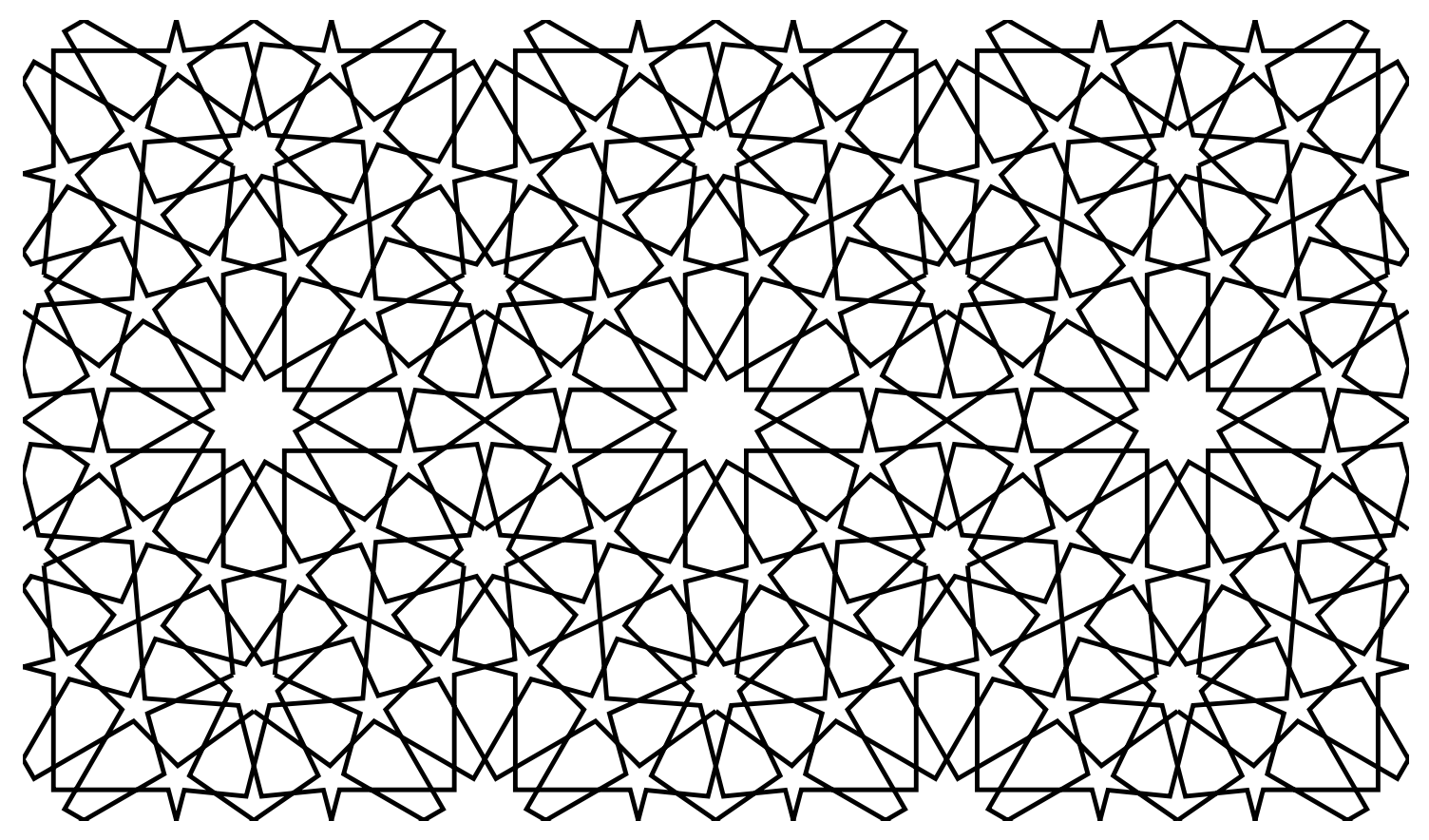

Figure 5: The sweeping curves seen flowing through this Seljuk design are produced by interpolating short straight line segments.

about the environment, which is transmuted into appropriate action. However, some bits of data are more useful than others. I shall use the term regularities ${ }^{2}$ for those features in the data that we process fluently, that is features for which we have developed fast, mostly automatic processing. Vision scientists have probed the visual system empirically and identified regularities based on reported preferences, response time measurements or, more recently, neurological imaging. The early Gestalt psychologists identified principles for grouping and relationships (proximity, similarity, continuation, symmetry and so on) [69], and we now have candidates for many more [61]. Some artists have also conducted methodical experiments to find combinations of elements that produce interesting responses, and developed their own design principles and rules of composition.

Besides fuzzing the visual system, we can also consider which structures and properties of the 3-dimensional environment survive in a usable form in a 2-dimensional projection, and are invariant under a range of viewpoints. Any features robust enough to meet these criteria would form a reliable basis for understanding visual data and therefore might have dedicated processing. Projection preserves qualitative properties such as continuity, connectedness, incidence structure (corners, intersections, and points of contact), convergence, straightness and alignment, but not metric properties (lengths or angles), parallels or symmetry. While mathematics shows these features make a reliable foundation for vision, this does not mean the visual system follows the rules of projective geometry to determine shape [26, 44].

Visual processing evolved to deal with the natural world, where many important struc-

\footnotetext{
${ }^{2}$ Regularity is one of the fundamental concepts of perceptual organisation, yet it has no agreed definition. In many cases, it refers to structures or constraints in the viewed environment, structures in the image data, or properties of the image data such as compressibility or complexity, all of which are independent of the viewer.
} 
tural features have biological origins [56]: radial growth from a point (flowers), growth along a line (leaves), expansion (spirals and cones in shells or horns), branch points or junctions (trees, antlers, veins in leaves), rhythmic repetition (centipedes, leaves along a stem). The structure of body plans is influenced by the number of degrees of motion: small floating things are roughly spherical, things that distinguish top and bottom have a radial symmetry axis, and things that have both an up-down axis and a front-back axis have bilateral symmetry. Although growth-along-a-line and bilateral symmetry appear similar, they arise from different causes: the first originates in spreading from a common spine, the second emerges when interaction with the environment induces symmetry breaking. The two cases have different properties: the spine may be a visible feature, it need not be straight, and the growth may be uneven, whereas the mirror line is an imaginary feature and the reflected halves will match quite closely. There is no a priori reason that information derived from the two situations should be equally useful or that the visual processing of the two cases should be the same. It is clear, however, that bilateral symmetry with a vertical mirror is special as it is detected much faster than other symmetries [66].

Some aspects of visual processing seem to be adapted to properties of the environment. For example, naturally occurring individual items rarely come in mirror-image pairs and, even if such things are found, they are not situated to display that symmetry unless there is an underlying cause - a mirror line is a property of a single object. Likewise, repetition (except in segmented animals like centipedes) is a property of multiple objects. Experiments indicate [12] that our processing is matched to this likelihood of occurrence: mirror images are recognised more quickly when the two parts are close together, and repeats are found more quickly when the objects are separated?

More subtle statistical properties of the environment have recently been applied to explain some counterintuitive properties of perception. Early experiments in psychophysics in the nineteenth century discovered that perceptions of physical properties are not absolute, but are biased by their surroundings: the same physical stimulus can generate different percepts in different contexts. These results are not an anomaly, but are a consistent, systematic distortion of reality. For example, the perceived brightness of an area with a given physical luminance depends on the context provided by the shade of neighbouring areas. The same is true for colour. Geometric primitives based on the comparison of lengths or orientations of line segments also exhibit this behaviour. For example, angles (the relative orientations of lines) are over- or under-estimated so that they appear nearer to perpendicular. All of these cases are based on relative rather than absolute values comparisons are made between different areas of the image. Purves and his colleagues have found that the discrepancy between real and perceived values can be predicted using cumulative frequency analysis that is calibrated with comparisons made in patches cut from images of the natural environment. He argues that such empirical statistics could have become innate in our visual system through natural selection, or could be learned or updated through experience. Note that the same general process, what Purves calls wholly empirical perception, applies to a variety of different physical attributes [47, 48]. This approach also explains several well-known visual illusions in which the perception of line configurations does not match reality [32].

\footnotetext{
${ }^{3}$ Approaches based on information theory and measures of symmetry find this hard to explain.

${ }^{4}$ Making decisions using empirical frequency tables is not the same as inference based on Bayesian probability models [19].
} 


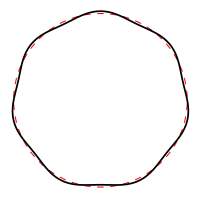

(a)

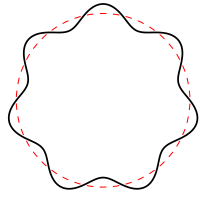

(b)

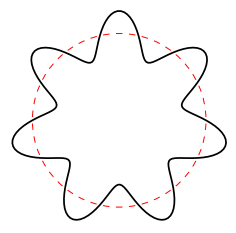

(c)

Figure 6: Shapes with 7-fold symmetry defined by radial frequency contours.

There is, of course, no reason that our internal representation of the environment has to be complete or faithful. It can be any abstract summary that is fast enough and accurate enough to be useful (have survival value). In any system with limited resources fast and accurate are likely to be competing requirements, and their priority will depend on the application: watching for danger has to be fast and will tolerate a higher false alarm rate than finding food.

We have seen that our perception of metric properties (length and angles) deviates from reality. The act of grouping also changes the perception of distance: items in a group appear closer than they really are [13]. So perhaps it is not surprising to find that the geometry of perceptual space is not Euclidean (another quirk of the visual system discovered by the pioneers of psychophysics). Perceptual space does, however, seem to be consistent with affine geometry so that properties like straightness, betweenness, collinearity, parallels, and convexity behave as expected $[57,60,62]$.

The Islamic geometric patterns we study are composed of straight lines. Our judgement of straightness has remarkable precision, even though it is not a commonplace feature of the natural world and we have no inducement to give it special treatment. The sensory data can be modelled by fitting an imaginary line using least squares regression; if the data form a small bump on either side of this line then the line will be perceived as not straight. In experiments [65], a line with a bump was discriminated from a straight line at a detection threshold finer than the spatial uncertainty due to the cone spacing in the fovea - a phenomenon known as hyperacuity. To achieve this 'subpixel resolution' the visual system presumably pools data from different viewpoints as the eye moves. Visual processing must also compensate for imperfections of the eye: wide angle optics are nonlinear, the axes of the various optical elements are off-centre and misaligned, the optical surfaces are asymmetric rather than surfaces of revolution, and the density of cones on the retina is very uneven [41]. Given these handicaps it is, as noted above, remarkable precision.

We can also detect deviations from circularity at hyperacuity thresholds [71]. A circle expressed in polar coordinates $(r, \theta)$ can be deformed by modulating its radius with a sine function

$$
r(\theta)=r(1+A \sin (n \theta))
$$

where $n \in \mathbb{N}$ is the radial frequency and $0<A<1$ is the amplitude. When $n>2$ deviations can be detected at very small amplitudes. Figure 6 shows examples of radial frequency contours that are noticeably non-circular. They are of interest here as a proxy for stars: when $n<7$ the curves can be identified after very short exposure times, but discrimination between larger frequencies falls off quickly after this.

This brief overview has covered properties of perception that we shall use to explore 
the regularities present in Islamic geometric patterns. We have seen that in the visual system affine properties are more salient than Euclidean ones. Thus straightness and good alignment matter more than accurate angles. We shall also see that alignment of imaginary abstract features like mirror lines seems to be important. Although symmetry plays a major role in patterns, it has not been a central topic in this survey. This is partly because little work seems to have been done on the perception of $n$-fold symmetry with $n>4$, and partly because the standard definition of symmetry based on isometries is part of Euclidean geometry, and we shall see that this is not sufficient to include all the examples we shall discuss.

\section{Accident or design?}

In the analysis of the tiled panel in $\S 2$, significant information was found in relationships that are unlikely to arise by chance, and the same principle is applied to perception. Such relationships are called non-accidental. 'Non-accidental' is not the same as 'unlikely' (statistically rare in the environment). It means that the relationship is a surprise or an anomaly, and 'not by chance' implies there is an underlying cause.

Building a visual system on features that are projective invariants ensures a high truepositive rate: a T-junction in the world reliably produces a T-junction in the image. However, while projection preserves incidence structure, it can also create false conjunctions: a T-junction in the image may correspond to a T-junction in the world, an occlusion where a line passes behind a surface, or a fortuitous alignment of unattached unrelated lines. Ignoring relationships that arise by chance reduces the false-positive rate.

Being able to identify non-accidental relationships relies on two principles:

- Uniformity hypothesis. There is order in Nature independent of any observer. This metaphysical assertion means that the viewed environment has a consistent statistical structure, and that repeated patterns of co-occurrence can be used to make predictions and find anomalies.

- Genericity hypothesis. The visual system solves the typical case and assumes that the situation is not exceptional in any way - the structural properties of the image will not change significantly if the 3 -dimensional arrangement is perturbed. In geometry, this is known as general position: if the key points (corners and endpoints) that define a configuration are moved slightly then existing points of contact and intersection will all be preserved, and no new ones will be created. In vision, the position of the observer is included in the configuration, so coincidental relationships cannot be introduced by a careful choice of viewpoint. Thus, this hypothesis subsumes the generic viewpoint hypothesis $[2,20]$, which is often singled out. A configuration that is in general position is called stable.

Many properties of our environment have physical causes. The laws of physics govern the way that light is absorbed and reflected by surfaces: smooth changes in the curvature

\footnotetext{
${ }^{5}$ Witkin and Tenenbaum [72] and Lowe [39] also draw parallels between perceptual organisation and the use of non-accidental relationships to discover causal explanations.

${ }^{6}$ A detailed account of what makes features reliable and useful can be found in [33].
} 
or orientation of a surface lead to smooth gradients in image textures. Edges, the discontinuities between homogeneous image regions, are used in the visual processing of both birds and humans, suggesting that the detection of these non-accidental features plays a key role in perception. In particular, the junctions between edges (corners and vertices) are used to identify objects [22]. At the human scale, matter is continuous and objects are connected. Some non-accidental relationships in the 2-dimensional image exist because the related image parts correspond to a single 3-dimensional object in the viewed environment. Such relationships can be based on geometric properties (parallel, collinear, smooth continuation), topological properties (converging, branching, closure) or shared attributes, including common motion. In these examples from early visual processing it seems that many non-accidental relationships (features bearing useful information) coincide with regularities (features we process fluently).

Associating causes with relationships in geometric patterns is not so straightforward? Ornament, particularly a pattern composed of straight lines, is a mark in the environment that is easy to discriminate from nature and conspicuously signals a human cause. In this sense, it is not accidental. However, its features result from a variety of underlying causes: cultural constraints of convention or style, structural constraints forced by the laws of geometry, the limitations of the medium, the practicalities of manufacture or reproduction, creative decisions made by the artist, or simply serendipitous by-products of these or other processes. Although these causes are not independent, we shall try to recognise their individual effects and, in particular, to identify aesthetic choices of the artist.

Let us look at some examples. Consider the light blue compartments in Figure 2. Together with the adjacent house-shaped pentagons, they are fillers that occupy the space left between the two primary motifs. The fact that the light blue compartments are bilateral is a consequence of the method - any shape whose interior meets the edge of the template is automatically joined to its reflection when the template is replicated. The pentagon, on the other hand, is what remains after all the other design decisions have been made. In strict geometrical terms, this residual space is asymmetric. However, it has approximate bilateral symmetry and is a deformation of an obvious ideal symmetrical prototype. Our perception of the shape will move towards or away from the ideal form depending on context, expectation, and how imperfect it is [21]. In this case, the artist has reinforced the bilateral interpretation by the symmetry of its floral decoration.

The pattern in Figure 5 has 9-pointed and 12-pointed stars as its primary motifs connected by a matrix of smaller 5-pointed stars. The distribution of the primary stars is governed by the $p 6 m$ symmetry type: the 12-pointed and 9-pointed stars are centred on the 6 -fold and 3-fold rotation centres, respectively. In patterns of this kind, the relative sizes of all the stars are determined by the packing of their circumcircles - another geometric control. The smooth curves seen flowing through this composition are by-products of this process. In fact, these illusory curves are an emergent property created by interpolating between the straight line segments forming the stars. During the experimental phase of the design process, the curves may not be so prominent, but an artist who notices the opportunity can enhance the curves by tuning the construction parameters of the pattern, such as the spikiness of the stars, to ensure that line segments are aligned to encourage interpolation

\footnotetext{
${ }^{7}$ If you start with enough data points, some striking alignments will occur by chance. This has been demonstrated with some 'surprising coincidences' in the configurations of neolithic standing stones $[8,36]$ and Woolworths stores [43].
} 
across the gaps. The fact that, in this case, the resulting curves flow uninterrupted across the pattern, producing one of its most striking features, is fortuitous.

Creating a new pattern involves choosing motifs, arranging them, and some iterations of adjusting, selecting and discarding, continually making judgements about aesthetic value to improve the composition. There are many factors that contribute to our aesthetic response. The fluency hypothesis [51] asserts that successful visual processing itself generates a reward. Even though our visual system evolved to process images of the natural world, if we get pleasure from structures that are easily processed then regularities are pertinent to art, too.

However, creating great art is not as simple as putting lots of regularities together and tuning the result to produce a super-stimulus. Even within the category of Islamic geometric ornament, some patterns look more stiff and lifeless than others. Excessive symmetry is one of the regularities that contributes to this effect. Regular repetition of a motif that is too simple, in music or ornament, quickly becomes tiresome, and a bit of jitter or variation is required to relieve the monotony. It is interesting to observe that some Islamic patterns, particularly from the Seljuk period, would not exist if their creators were concerned about mathematical correctness. Indeed, some patterns appear to show geometrically impossible arrangements of incompatible elements. Documents of the period (see [42] or [53] for example) contain critical remarks by mathematicians about the poor geometric reasoning skills of artisans, who were satisfied with approximations that produced the visual effect they desired. We shall see examples in $\S 8$.

\section{Visual ambiguity}

Islamic geometric ornament is composed of flat linear figures, a trivial example of which is shown in Figure 7(a). Even such a rudimentary figure can be interpreted in multiple ways, for example as overlapping square frames, or as two (non-convex) hexagons pushed together - the component parts of both situations are shown separated in (b). In the first interpretation, the squares must be either skeletal frames or transparent because they do not occlude one another in (a). To describe two squares we can list the coordinates of the eight corners; we do not need to specify the intersection points in the figure as they are consequences of other information. To describe the two hexagons we list the coordinates of twelve corners, and note that the polygons are placed so that two pairs of coordinates coincide.

The genericity hypothesis is defined in terms of a viewer in a 3-dimensional environment. Although our figures of interest are 2-dimensional, we can apply analogous arguments here: recall a configuration is stable if its structural properties do not change when it is perturbed slightly. Figure $7(\mathrm{c})$ shows the two cases after the defining vertices have been perturbed: the two 4-sided polygons still overlap and yield two transverse intersection points, while the coincident vertices of the two hexagons can become disconnected or generate a new overlap. The two-squares interpretation is stable, and the contrived (non-accidental) nature of the two-hexagons configuration becomes immediately apparent. It is well-known that experiments show people prefer the two-squares interpretation in general.

Figure 7(a) can be embellished to reinforce either interpretation. Two options are shown in (d): the squares are treated as interlaced ribbons, which encourages a line-based interpre-

\footnotetext{
${ }^{8}$ Standard explanations claim that the two squares interpretation is 'more likely' or 'simpler', depending on the stance of the author.
} 

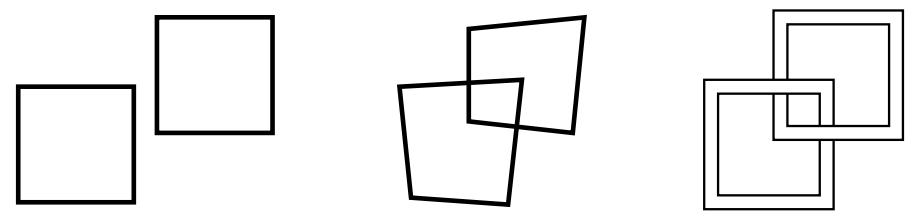

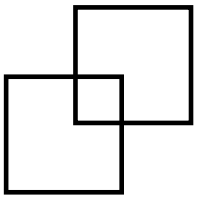

(a)

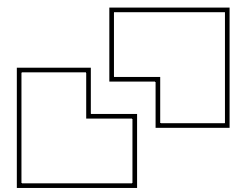

(b)

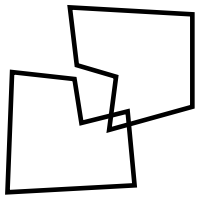

(c)

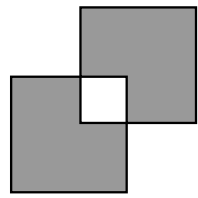

(d)

Figure 7: Interpretations of a diagram.

tation of the figure; the hexagons are filled with a solid colour, which leads to a shape-based interpretation of foreground forms against a white background. Both treatments are used in Islamic designs. Ribbons are carved in stone, plaster or wood. The shape-based approach is implemented with glazed ceramic tiles in a range of colours, which are cut to size and abutted to form a continuous surface. When regions of a pattern are filled with colour, the shapes become the primary objects and the lines become borders that no longer have an independent existence.

Interlacing is an abstract stylistic treatment applied to a line-based design as a visual effect. The ribbons are an emergent property, not the primary objects brought together by the artist during the creative process. In most examples of Islamic interlaced ornament the sequence of over- and under-crossings alternates when you follow any ribbon. The lines in any pattern that is planar and 4-valent can be transformed into ribbons with this alternating weave - a discovery that has been made by many cultures that use geometric ornament. In fact, the alternating property is so ubiquitous that it is often assumed to be a mistake by the maker when the ribbons fail to follow this rule.

Interlaced patterns introduce new perceptual problems. We are presented with a set of lines and shapes in a plane, yet we see continuous ribbons passing in front of and behind one another. There is rarely any attempt at realism in the stimulus: depth cues such as shading are absent, and the ribbon surfaces at a crossing are not sculpted to be at different heights. T-junctions and continuity of line provide strong enough cues to occlusion that a simple arrangement of lines is sufficient to create the layering effect.

Except in very simple cases, interlaced ornament does not depict 3-dimensional objects. Even when it appears to do so, we may be deceived and be looking at an impossible object. Figure 8(a) shows an arrangement of three circular rings linked together with an alternating weave. This design has been used for centuries to symbolise strength in unity (remove any ring and the object falls apart) [17]. The mental model suggested by this image may be something like paper strips interwoven to produce a flat object of negligible thickness. Trying to give it a more 3-dimensional interpretation causes problems: no arrangement of planar circles in space can be linked in this fashion, whatever their relative sizes or orientation [38]. Figure 8(b) shows a similar arrangement of three rings carved in relief the over-under rule for interlacing has been broken to preserve the flatness of the rings.

The brightly coloured polygonal cells in Figure 2 are decorated with floral arabesques. 


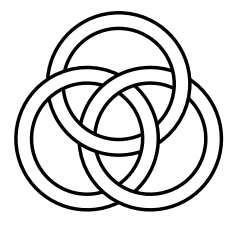

(a)

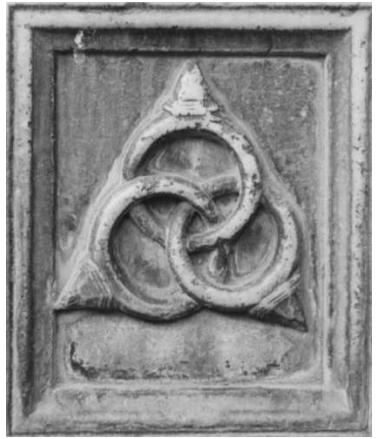

(b)

Figure 8: The drawing of three interlaced circular rings depicts an impossible object. When carved in relief, the interlacing is altered. (b) is from the Sforza Castle, Milan.

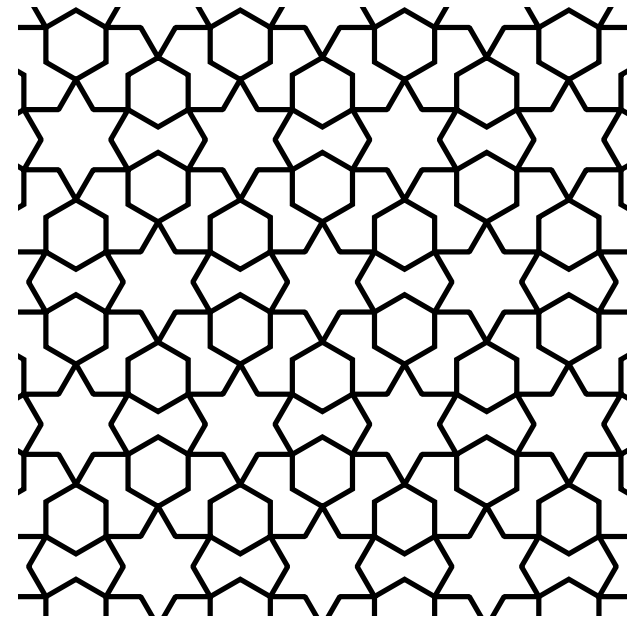

(a)

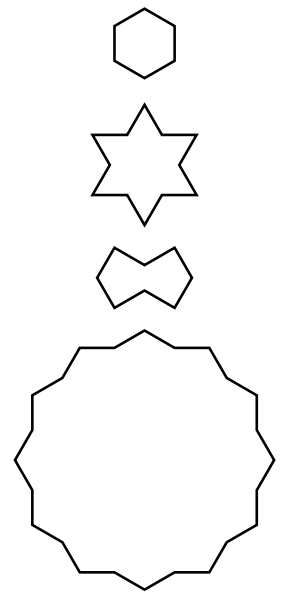

(b)

Figure 9: A design with three kinds of compartment, and one kind of component. 
Filling the contours helps the individual shapes function as coherent units, and gives them an identity that can be recognised in copies through the design. This, in turn, leads to the impression that the panel is an assembly of interlocking pieces, like a jigsaw puzzle. Some Islamic patterns are composed in this way using a modular design system: a small set of shapes provides the raw ingredients for composition, and patterns are created by selecting and arranging the shapes different ways [15]. Each design system produces a family of related patterns; the same shapes appear in different configurations within a single pattern, and they are reused in multiple patterns. However, Figure 2 was not devised this way: some shapes are unique to this pattern and, as we saw in $\S 2$, the shapes are an emergent property that arise after the development of a line-based doodle induces a fragmentation of the plane. Just as ribbons are not the primary motifs arranged by the artist, so focussing on shapes can also divert attention away from the design process, and care is required when looking for evidence of design decisions.

We need to be able to refer to line-based and shaped-based elements of patterns. A compartment is a connected region of the plane bounded by lines of the figure. In a 4valent figure, a component is the set of lines traced out by travelling directly on at each vertex, not turning right or left. The pattern in Figure 9(a) shows that the 'travel directly on' instruction need not be 'straight ahead'. In this example there are three kinds of compartments: regular hexagons, 6-pointed stars, and bone-shaped octagons. All the components are 12-pointed stars. This pattern was probably composed by arranging the regular hexagons and stars, with the octagons emerging as background fillers. The component view is unusual in that the components intersect at their vertices, rather than having crossing edges. In this case, both interpretations involve an unstable configuration with coincident vertices.

Components are often unplanned features that emerge after primary motifs have been placed together in a composition. Some components are easily perceived, particularly if they are small, convex and fairly symmetrical or familiar (as in Figure 1); small adjustments can be made to a pattern to enhance these properties and make the components more prominent. Figure 10 shows that components can be closed or unbounded. It also demonstrates that a closed component may be too large to fit in any section of a pattern that would be used as decoration [29].

Our final look at ambiguity concerns hierarchical structure and higher levels of organisation. The pattern in Figure 11(a) has compartments of three shapes: a square, a house-shaped pentagon, and a bone-shaped octagon. It is an example of a field pattern (it has no stars). Many of the vertices are 3-valent so it does not have a well-defined set of components. This small-scale pattern is rather poor, having several monotonous patches where the local configurations consist of a single shape - four squares together, or four houses. The redeeming feature of the design comes from the variety of larger-scale patterns that can be found within it. Some examples are highlighted in blue in the other parts of the figure. These embedded patterns are simple, easy to recognise, and early: (b) and (c) are found together in the ninth-century al-Tariq Mosque in Balkh, and (d) is an Archimedean tiling used as a floor pattern in Classical times. Notice that the three designs do not have any edges in common and could be overlaid simultaneously in different colours. It is not obvious to the viewer that this design is a puzzle, that it presents a challenge to explore and make sense of it. The unexpected discovery of familiar patterns hidden within it results in an 'Aha' moment, which in itself is a source of aesthetic pleasure [40]. 


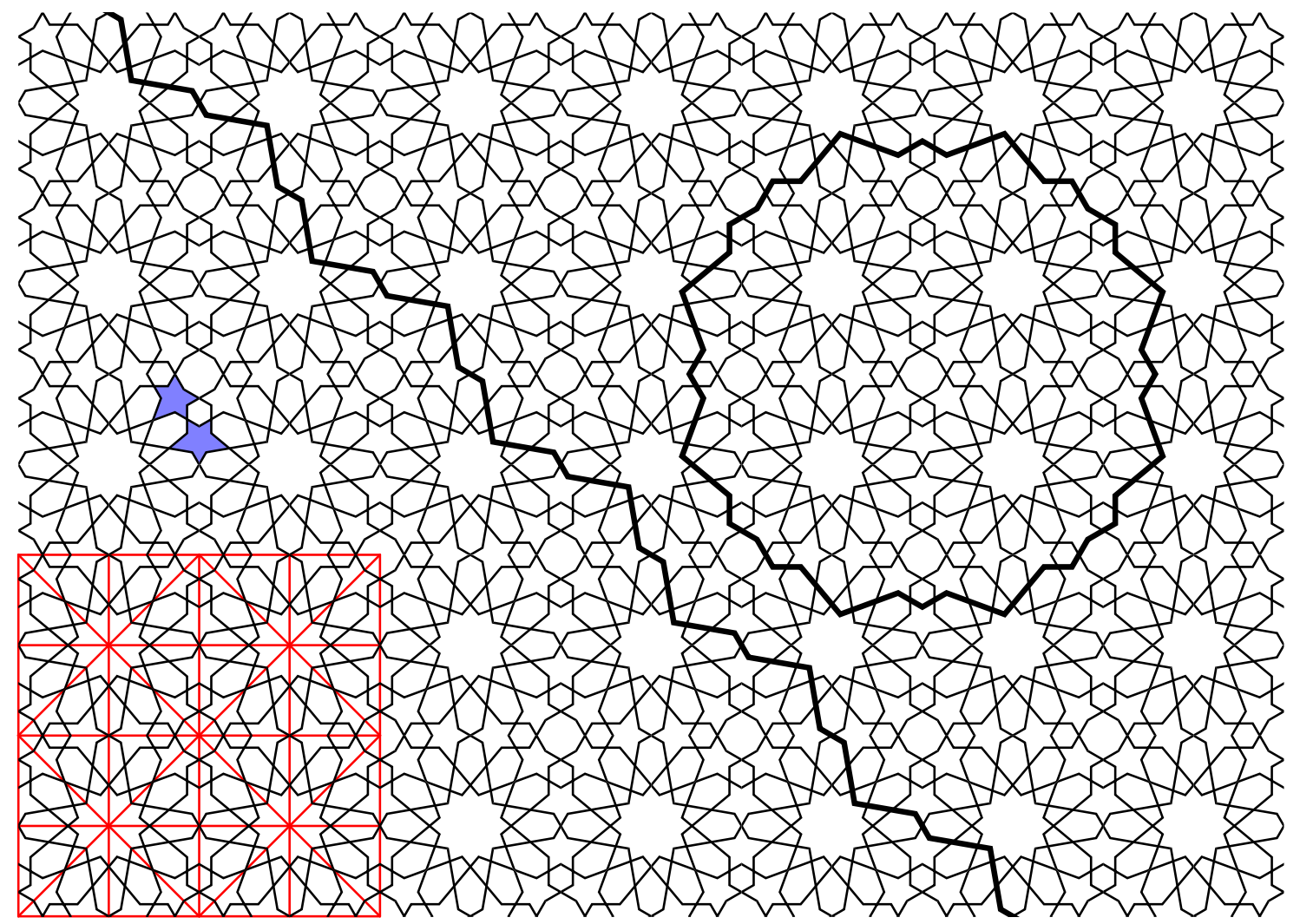

Figure 10: Components can be closed or unbounded. They can also be so large that they may not appear whole in any implementation. 


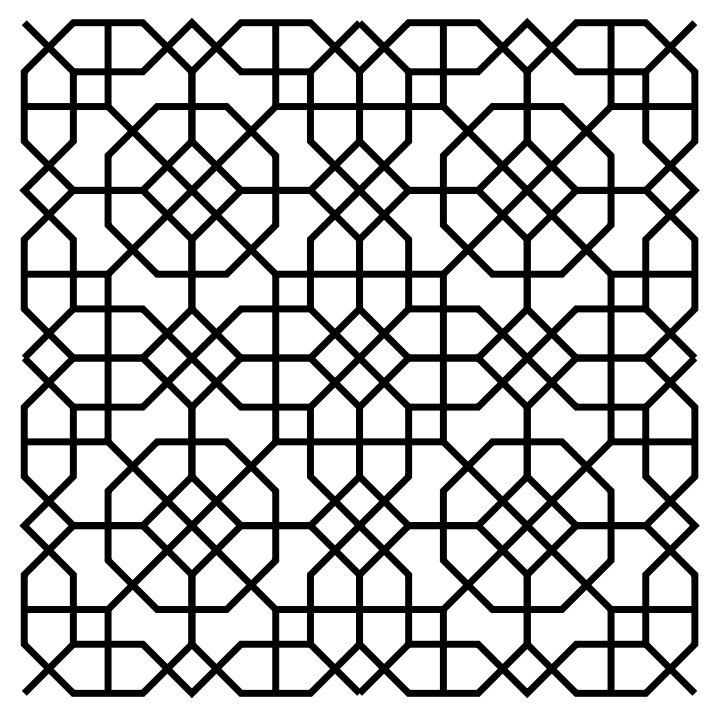

(a)

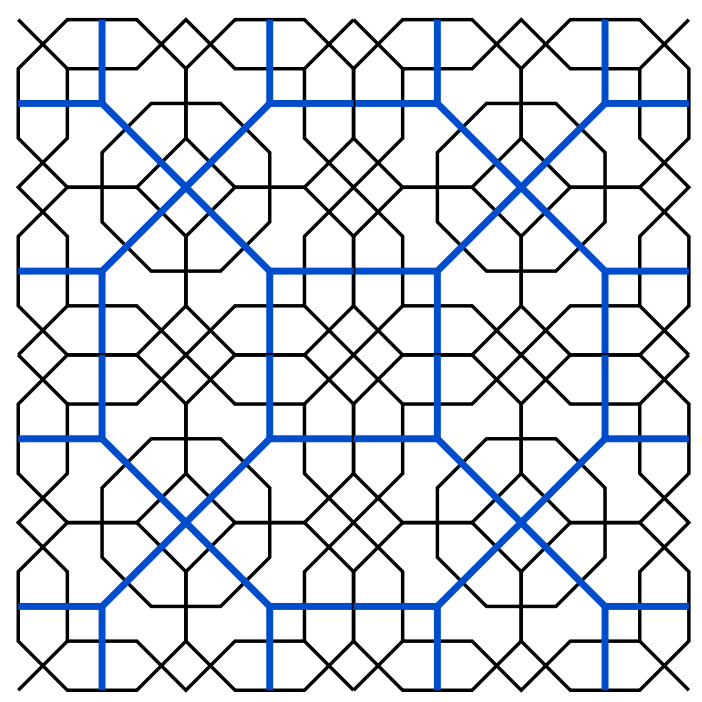

(c)

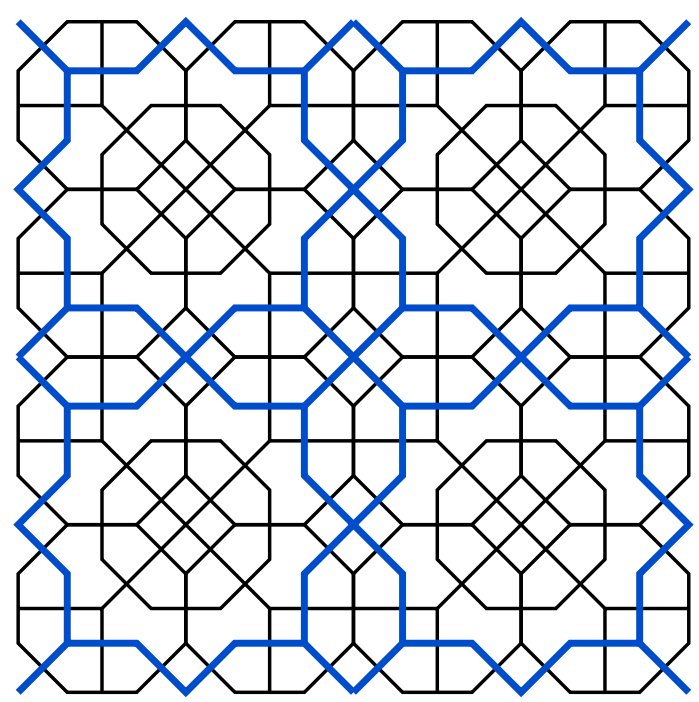

(b)

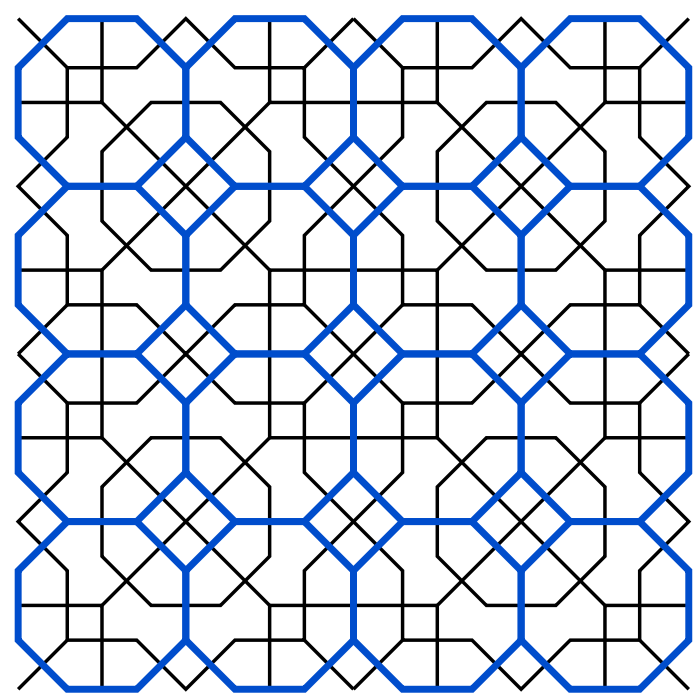

(d)

Figure 11: This Mughal pattern can be perceived in multiple ways. 


\section{$6 \quad$ Finding structure in star patterns}

In our analysis of Figure 2 we found structure on different levels. A top-down analysis identified a small template that could be replicated to lay out the whole pattern, and a more bottom-up approach focussed on the motifs and how they were brought together to form the composition. We can continue this two-pronged strategy to find more general principles for the perception and composition of Islamic patterns.

\subsection{Large-scale order: repetition by reflections}

Most Islamic patterns have mirror symmetry. Drawing all the mirror lines on a pattern divides it up into regions, each of which can be used as a template to regenerate the whole pattern. So long as the mirror lines are not all parallel, the regions will be finite. The four possible arrangements of mirror lines leading to finite regions are shown in Figure 12. The left-handed and right-handed tiles are differentiated by shading. The tiles are marked with L-shaped motifs to destroy some of their inherent symmetry; in (d), the tiles themselves are asymmetrical so no marks are required. In practice, the tiles will be decorated with fragments of the pattern.

In these cases, the markings are chosen to produce asymmetrical tiles; the resulting symmetry types ${ }^{9}$ are called primitive. The tiles could also be marked with motifs that have rotational symmetry - the possibilities are shown in Figure 13. The rectangular tiles with primitive type $\mathrm{pmm}$ give rise to two other arrangements: a motif with 2-fold rotational symmetry produces a design with $\mathrm{cmm}$ symmetry type; if the rectangular tile is made square, the motif may have 4-fold symmetry and this leads to type $p 4 g$. If the equilateral triangle tiles in primitive type $p 3 m 1$ carry a motif with 3 -fold symmetry, the design will have $p 31 m$ symmetry type.

In one approach to understanding symmetry groups [11], rotation centres that do not lie on a mirror line are called gyration points. A rotation centre that lies at the intersection of several mirror lines is forced and carries no additional information - the rotational symmetries are a mathematical consequence of the mirrors. The presence of gyration points, on the other hand, adds variety to the structural ${ }^{10}$ relationships within a pattern, possibly making it more interesting, and is a choice of the artist.

From the geometric viewpoint, the symmetry properties of repeating patterns are well understood. The mathematical theory was developed at the end of the nineteenth century to understand the 3-dimensional periodic structures of crystals. Later, the techniques were applied to 2-dimensional decorative ornament to enumerate symmetry types of strip patterns, wallpaper patterns, layered patterns, and so on. Scholars from a range of disciplines have studied decorative art from across the world and found that artists have created patterns that exhibit many of these types. However, identifying symmetry types in the field is not always straightforward. Real patterns are not geometrically perfect so some tolerance

\footnotetext{
${ }^{9}$ Patterns that repeat in two directions have one of 17 symmetry types. The labels used here follow the nomenclature used by the International Union for Crystallography in its tables. Symmetry types of many Islamic patterns are illustrated in [1], although readers should be aware that some of the types are wrongly identified (this can be the case, for example, when the template used by the authors carries a motif that has non-trivial symmetry).

${ }^{10}$ The two types are mathematically distinct: gyration points correspond to local cyclic subgroups $C_{n}$ and the rotation points on mirrors to local dihedral subgroups $D_{n}$.
} 


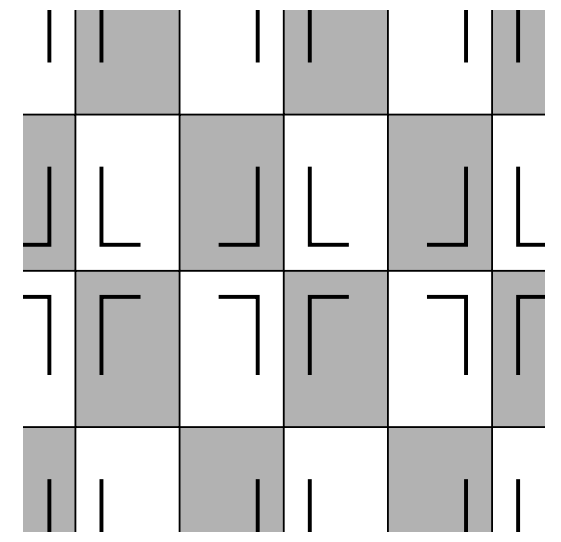

(a) $p m m$

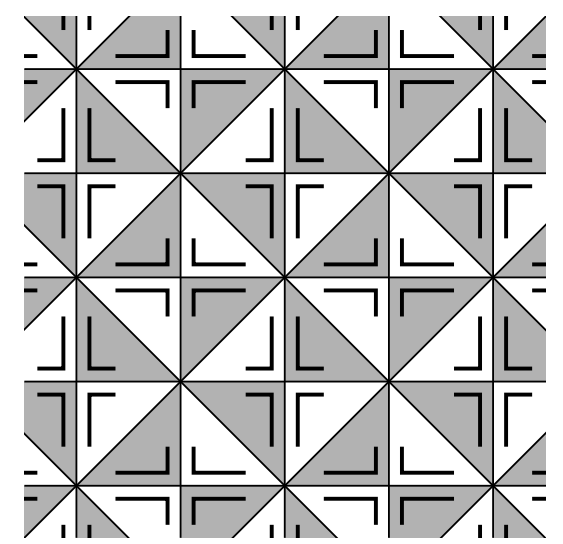

(c) $p 4 m$

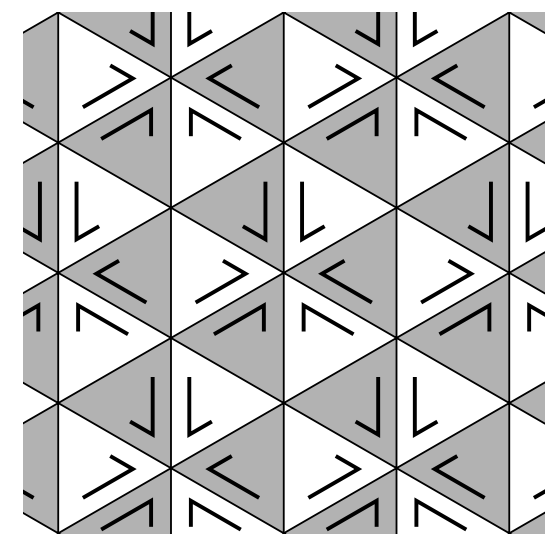

(b) $p 3 m 1$

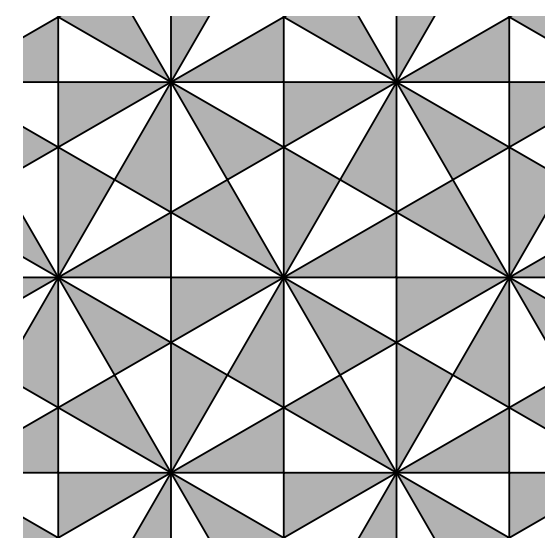

(d) $p 6 \mathrm{~m}$

Figure 12: The tilings generated by reflections in the sides of a tile with an asymmetric motif. 


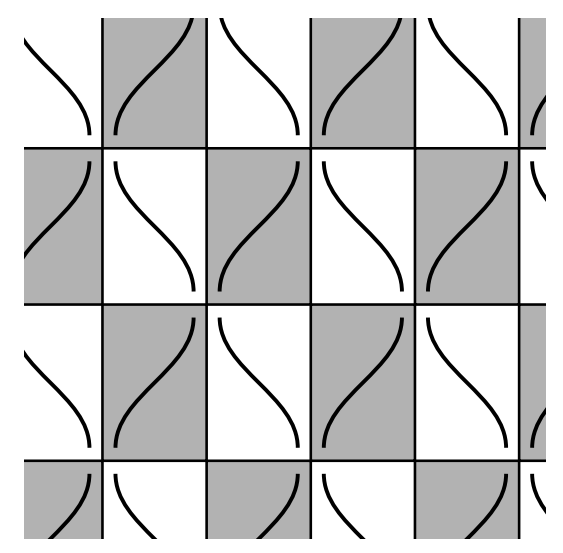

(a) $\mathrm{cmm}$

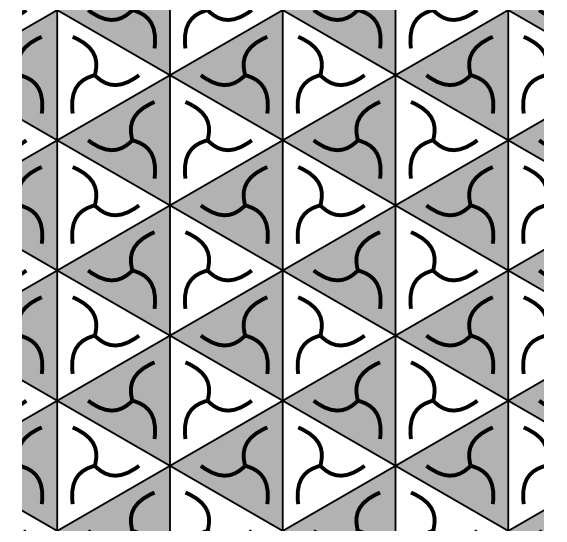

(b) $p 31 m$

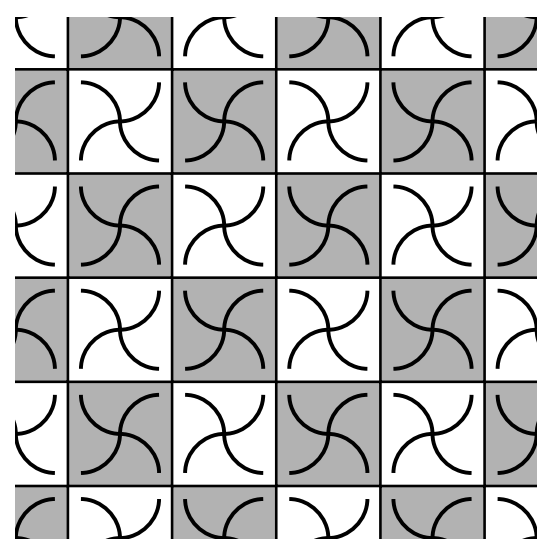

(c) $p 4 g$

Figure 13: The tilings generated by reflections in the sides of a tile with a rotationally symmetric motif. 
is required for matching and alignment. If the pattern is interlaced, should we use 2-sided patterns or ignore the crossings? What about colours? Inconsistencies in such choices between, and even within, analyses means that mathematicians do not agree on which of the 17 wallpaper types are represented by the large collection of Islamic patterns in the Alhambra [27].

Even if identifying a symmetry type can be more of an art than a science, it is still clear that the distribution of symmetry types used in practice is uneven and varies between cultures. A comparative study [6] of British and Egyptian preferences for symmetry in small geometric arrays found a higher ranking of mirror symmetry by Egyptian subjects, something which may simply be due to frequent exposure to Islamic geometric patterns in their everyday environment. Washburn argues that symmetry types are a cultural signature that can be used as an analytic tool in anthropology - the designation of types as traditional can be linked with asserting and maintaining group identity. She has found examples where the same motifs are used in different arrangements to differentiate neighbouring tribes [63], and to differentiate the products of a single group as being for their own use or trading with outsiders [64].

An absence of certain symmetry types need not imply that artists were aware of the possibilities and deliberately avoided them. Restrictions on the arrangement of motifs can also arise as indirect consequences of other stylistic or aesthetic choices. For example, the fact that the ribbons in Celtic interlaced ornament are closed and do not have free ends renders some 2-sided frieze types impossible [14]. The patterns illustrated in Figures 12 and 13 are generated by reflections in the sides of a finite region. This property has the following practical consequence: if the fundamental region is made into a stencil, the pattern can be marked out by turning the stencil over along its edges. Using this mechanism for laying out patterns means that only seven of the 17 symmetry types of periodic patterns can be produced.

In fact, only five ${ }^{11}$ of the seven types occur in traditional Islamic patterns: $p 3 m 1$ and $p 31 \mathrm{~m}$ are never used in star patterns. The absence of 3 -fold examples is not the result of a technical limitation: it is possible to create authentic looking star patterns in equilateral triangular templates. Aesthetic and practical considerations can explain this observation. Islamic patterns contain whole repeat units (unlike Roman patterns, for example, which can be crudely truncated when they reach the edge of the available space). Moreover, designs are usually applied to rectangular spaces. Satisfying both these criteria requires a rectangular template. The fundamental regions of $\mathrm{pmm}, \mathrm{cmm}$ and $\mathrm{p} 4 \mathrm{~g}$ are rectangular or square, in $p 4 m$ two triangular regions sharing a long edge can be joined to form a square, and six copies of the fundamental region in $p 6 \mathrm{~m}$ make a rectangle. (Medieval manuscripts such as the Topkapi Scroll contain rectangular templates of these composite forms.) In this format, a $p 6 \mathrm{~m}$ template resembles a $\mathrm{cmm}$ template: a rectangular panel with a 2 -fold rotation point in the middle. The fact that, in the generated pattern, two corners of the rectangle become 6 -fold centres rather than 2 -fold centres and a diagonal of the rectangle lies on a global mirror line is not apparent.

Mirror lines are salient features for visual processing, particularly if they are vertical or horizontal. Mathematically, there is no uncertainty about their existence or location, and they determine a unique, canonical decomposition of a pattern. We have seen that the

\footnotetext{
${ }^{11}$ Examples of the five types appear in the figures: $p m m$ Fig. 24, $p 4 m$ Figs. 10 and 11, $p 6 m$ Figs. 5 and 14, $\mathrm{cmm}$ Figs. 23 and 26, $\mathrm{p} 4 \mathrm{~g}$ Figs. 2 and 25.
} 
large-scale structure of star patterns is governed by mirror lines. Mirrors also function as natural break points, both geometrically and visually. The choice to use whole templates to form a decorative panel means that mirrors lie along the boundary of the panel, making it easy to imagine how the pattern could be continued.

\subsection{Hankin diagrams}

Mirror lines slice a pattern into sections suitable for use as templates, but these pieces are not the parts assembled by the artist to create the composition. Nor are they what we mean when we say we perceive the whole design as different from the sum of its parts. Global symmetry operations provide the rules for replicating a template, but they say nothing about the choice of motifs, or their local disposition. Can we identify salient parts in Islamic patterns, and do they correspond to the elements selected and arranged by the artist?

In Figure 2 the assembly of the two primary motifs is rather clumsy and we could detect the join quite easily. In general, it is much harder to find a unique division into coherent parts. By comparing lots of patterns, we can learn to identify common configurations that are re-used. These preformed motifs are self-contained and have an independent identity outside of patterns - they are elements that define the vocabulary of Islamic style. However, even without this knowledge and experience, people perceive structure in the polygonal clutter of an unfamiliar pattern. The low-level visual processing of the specialist and the uninitiated alike highlights the same generic regularities as worthy of further attention.

In this section we shall examine a method for annotating Islamic patterns that exposes structural properties. The technique is useful for analysis and, in rare cases, the resulting framework also forms part of a finished artwork.

The technique is illustrated in Figure 14 on patterns formed by placing regular 12-sided polygons on a triangular lattice. Variants of the pattern are produced by changing the size of the dodecagon components while maintaining the same centres and orientation. In (a) the components are scaled so that the crossings occur at the midpoints of their edges; all the compartments are equilateral.

Because the pattern is planar and 4-valent, the compartments can be coloured in two colours so that adjacent compartments have different colours. This has been done in Figure 14(b) using red and blue. We will produce two segmentations of the pattern, one for each colour. Choose a colour and at each crossing draw a line that bisects the angle of the chosen colour. The results for the blue and red choices are shown in (c) and (d), respectively. The blue bisectors can be extended until they meet each other, forming a connected network of rhombi, as shown in (e). Each rhombus tile is decorated with a bone-shaped octagonal compartment. Extending the red bisectors until they meet does not form a connected network - it just produces hexagons around the 6-pointed stars, as shown in (d). We can add extra lines between the corners to form a connected polygonal network, as shown in Figure 14(g), but the additional lines do not meet the pattern. This produces two kinds of polygon: regular hexagons decorated with 6-pointed stars, and isosceles hexagons decorated with what is called a shield motif. I shall call a pattern annotated with a polygonal grid like the examples in Figure 14(e-i) a Hankin diagram after E. H. Hankin, who used them in his analyses of Islamic patterns.

The grid in a Hankin diagram influences our perception of structure in the pattern in 


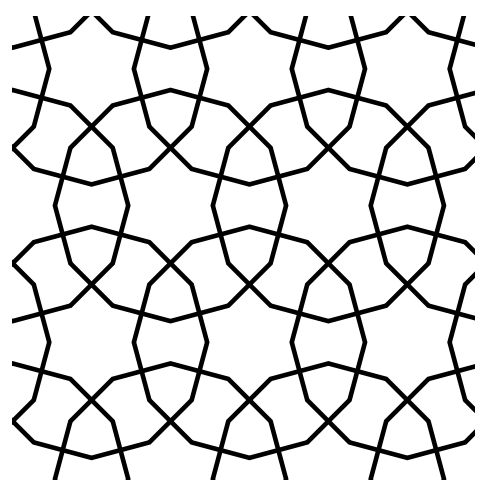

(a)

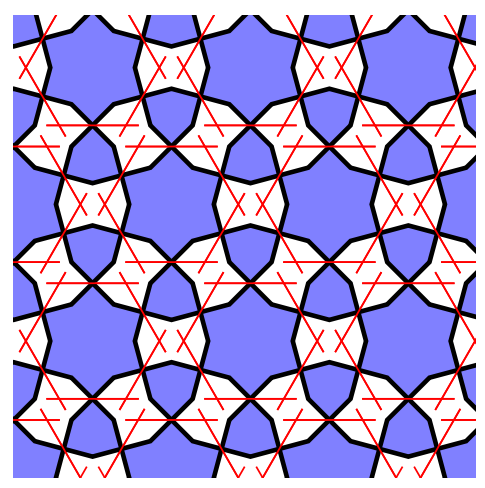

(d)

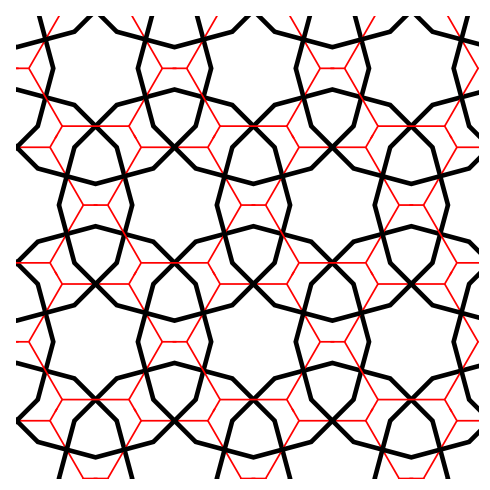

(g)

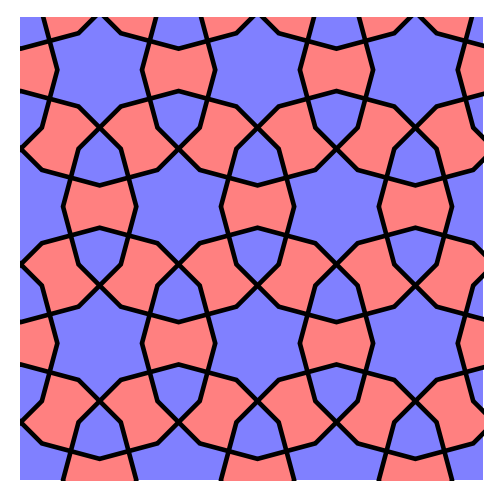

(b)

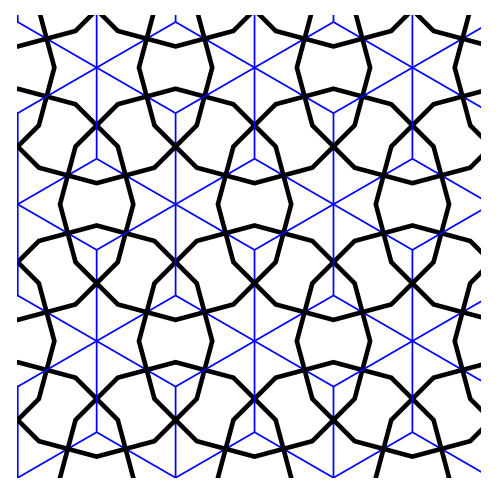

(e)

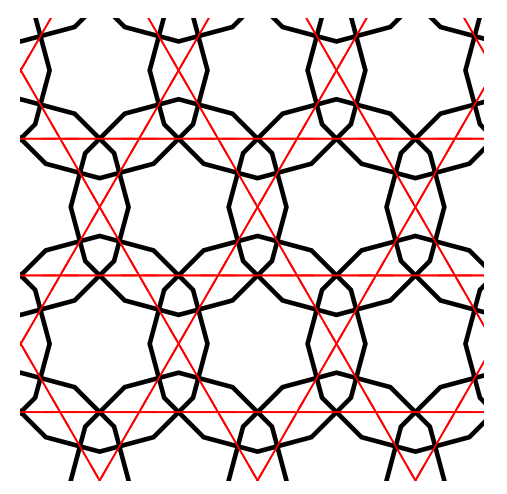

(h)

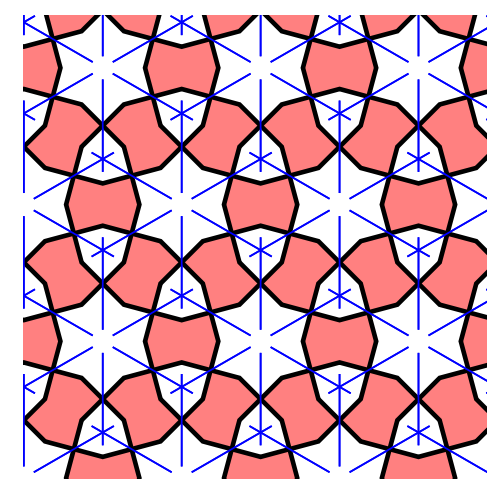

(c)

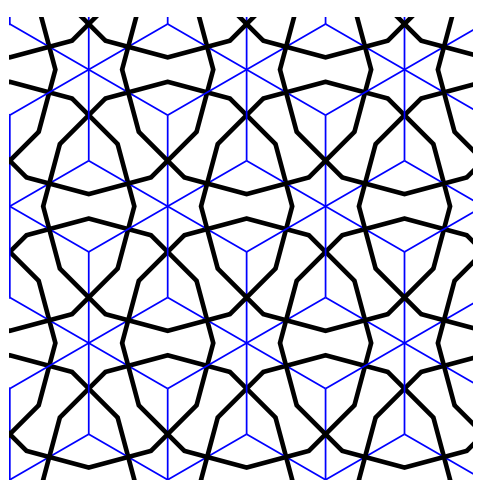

(f)

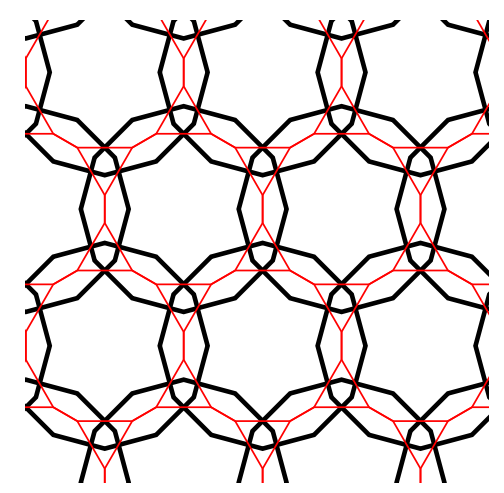

(i)

Figure 14: Constructions of Hankin diagrams. 
several ways. First, it divides the compartments into figure and ground. In Figure 14(g) what were the blue regions in (b) are enclosed in red polygonal zones and selected for our attention; they have become the primary motifs and hence are seen as foreground. The polygonal zones in the grid define a 'catchment area' for each motif. The shapes of the background regions are secondary, merely gaps. Indeed, if we regard the pattern as composed of the grid polygons, each decorated with a motif, then a background compartment is formed by fusing the corner regions of a patch of grid polygons - its shape is a by-product. Switching to the blue grid reverses the figure-ground relationship. Second, the grid makes the structure of the pattern much easier to describe. To identify pattern compartments in our earlier discussions, we resorted to references to familiar objects (house-shaped, boneshaped). The polygons generated by the bisectors are simpler, symmetric, often convex, and are familiar enough to have common names.

The grid in a Hankin diagram captures some of the structural properties of the pattern. For example, symmetry elements of the pattern such as mirror lines and rotation centres are inherited and shared by the grid. However, the relationship between a pattern and its grids is rather variable: significant geometric properties of the pattern need not be correlated with special configurations of the grid. In the following list we continue to use the examples from Figure 14.

- In (e) the crossings in the pattern lie on the blue edges about $36.6 \%$ of the way along. In (f) the components are scaled to cross the grid at the midpoints of the blue edges. This produces larger dodecagonal components, but there is nothing visually distinctive about the pattern.

- We might expect small changes to the pattern to induce small effects on the grids. In $(g-i)$ the components vary slightly in size, and the red grids have three different topologies. In (h) all of the grid edges meet the pattern, but in (i) as in (g) nugatory edges need to be added to form a connected grid.

- The pattern variants in $(\mathrm{e}-\mathrm{i})$ have a very strong symmetry property: the symmetry group of the pattern acts transitively on the crossings, which means that all the crossings play an identical role in the pattern. Therefore, edges in the grid that are generated directly from the crossings will all play equivalent roles in the grid. In particular, the incidence angles between the pattern and a grid are all equal, and the crossings lie at the same position along each grid edge. This is not typical of Islamic patterns in general.

- In (g) the special property of the pattern (equilateral compartments) does not produce any noticeable characteristic in the grid. Conversely, in (f) and (h) the special property of the grid (crossings at midpoints) is not noticeable in the pattern.

This method of decomposing Islamic patterns was first described by Hankin [30]. He records how he found two lines that passed through crossings scratched into a plaster ceiling in a bath house at Fatepur Sikri, India, and he assumed they were construction lines used to lay out the pattern. By imitating these lines at other crossings, he constructed polygonal grids like those described above. Although he does not mention them, some carved jali screens from the same site include a pattern and grid together in the form of a Hankin diagram - examples are shown in Figure 15. 


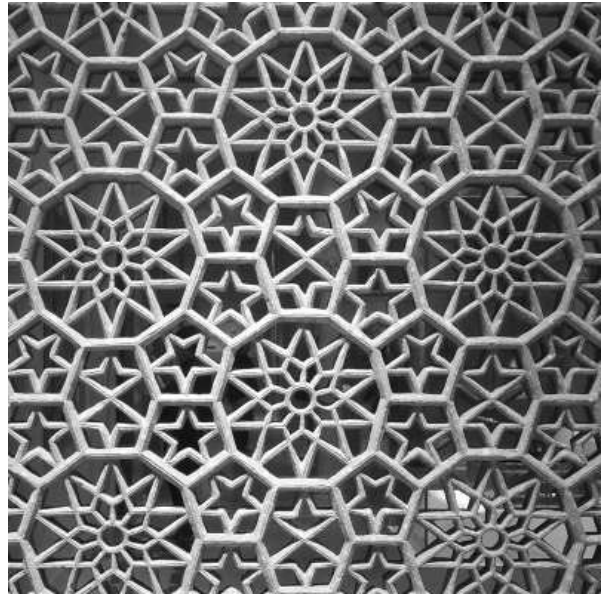

(a)

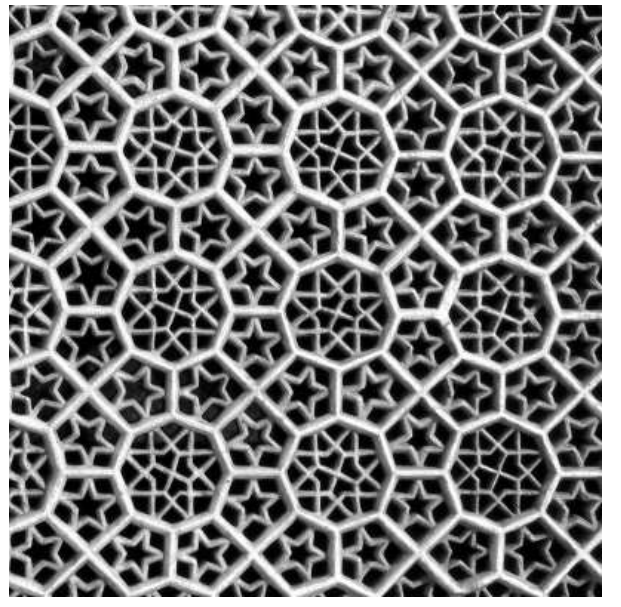

(b)

Figure 15: Sixteenth-century jali screens from Fatepur Sikri, India.

In the construction described above the grid is derived from the pattern, but Hankin thought the process was used in reverse: the grid provides a convenient intermediate step in the construction of the pattern, and could even be part of the creative process to design new patterns. While it may serve as an aide-mémoire and establish some constraints, the grid alone does not supply all the information required. When grids are constructed from historic patterns the crossings often do not lie at the midpoints of edges, the incidence angles may vary, and edges in the grid may not correspond to crossings in the pattern. Nevertheless, Hankin presented the following procedure (sometimes called 'polygons in contact' after a phrase in his paper) as a simple method for drawing Islamic geometric patterns: draw a polygonal grid, choose an incidence angle that will be common to all crossings, and draw crossing lines at the midpoint of each edge, extending them until they meet other such lines. The process is easy to explain (it appears on YouTube) and to implement on a computer $[34,35]$. In the next section we shall examine how it works in practice.

Hankin diagrams are helpful because they reveal order in complex data. Most Islamic patterns are 4-valent so the technique can be applied, and it identifies good candidates for perceptual and artistic parts. The segmentation process is difficult to perform consciously in the mind's eye, even with practice and experience of the likely results, so the diagrams are drawn as part of technical analysis. They decompose a pattern into units that can be thought of as polygonal tiles decorated with pieces of the pattern. Thus, the decomposition defines both a vocabulary (motifs on the tiles) and a grammar (arrangement of tiles) of the pattern; it abstracts both parts and relationships. Bonner [7] has drawn Hankin diagrams for many historical patterns and shown that there are families of patterns that share the same motifs but have different grid structures, and conversely there are families of patterns that have the same grid but different motifs.

When using Hankin diagrams in historical analysis, we must take care not to allow the choice of grid to influence our interpretation of the pattern. In Figure 14, depending on the argument we want to make, we may prefer a blue grid because the tiles are all congruent and all the edges meet the pattern, or a red grid because the tiles have higher-order symmetry and the pattern meets the grid at midpoints of its edges. There is also a temptation to 


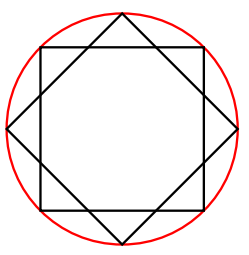

(a)

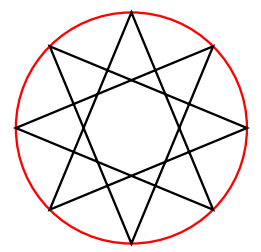

(b)

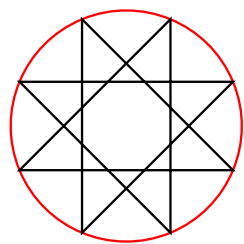

(c)

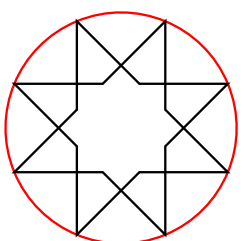

(d)

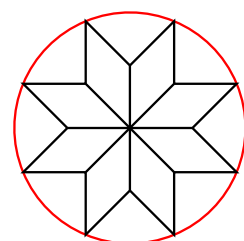

(e)

Figure 16: Some 8-pointed star motifs.

regard Figure 14(h) as the 'correct' form of this pattern because its grid has nice properties (all the grid edges meet the pattern, all the crossings lie at midpoints of the edges, all the tiles have 3 -fold or 6 -fold symmetry). However, we must not adapt the data to fit the model. It is likely that this simple pattern was conceived as overlapping dodecagons and the proportions used in (a) produce a nicely balanced design.

\section{$7 \quad$ What makes a good star?}

Star motifs are the quintessential elements that characterise Islamic geometric ornament. They take a variety of forms ranging from simple zig-zag polygons to more complex figures with a cellular internal structure. This makes it difficult to find a universal set of defining features. For example, the compartments highlighted in blue in Figure 10 are closed zig-zag shapes with five spikes and bilateral symmetry, but it is uncertain whether they would be recognised as stars without some prompting; they are residual spaces left after the primary motifs have been positioned. Compare them with the 5 -pointed stars in Figure 5 where the primary 9-pointed and 12-pointed stars and the smaller secondary stars are all constructed explicitly in their own right and are all well-formed.

Despite lacking a clear definition, we shall explore different constructions to create star motifs. These constructions are not the only ways to create star-like polygons, but they yield sufficiently varied examples to illustrate which properties are important. The polygons are all zig-zags that close up on themselves. Even so, some are more successful than others at capturing our notion of starness. In this section we investigate stars in isolation, and in the following section we see how to arrange them together to form a composition.

\subsection{Classical construction}

Early stars have very simple geometry and are easy to construct: place $n$ points equally spaced around a circle and connect every pair of points $d$ steps apart by a straight line. The resulting shape has a modern mathematical notation and is denoted by the symbol $\{n / d\}$. Figure $16(\mathrm{a})$ and (b) show $\{8 / 2\}$ and $\{8 / 3\}$, respectively. The use of these stars in geometric ornament predates the birth of Islam and examples can be found in Classical mosaics [4].

The stars in Figure 16(b) and (c) are congruent. Although they are geometrically identical, they have different visual and artistic properties: one is precariously balanced, the other is stable. Verticals and horizontals are salient features, both explicit forms such as lines and inferred forms such as mirrors. The vertical mirror line dictates the perceptual frame of reference for both these figures. In (b) it connects two spikes and in (c) it lies 


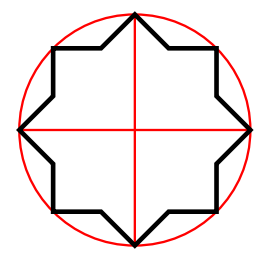

(a)

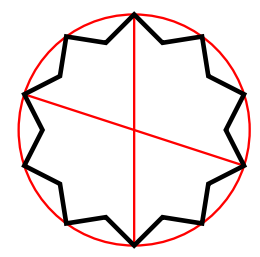

(b)

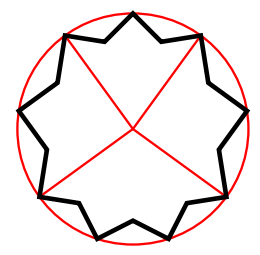

(c)

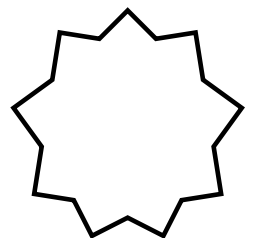

(d)

Figure 17: A cut-and-paste approach to star construction.

between two vertical lines, producing different descriptive schemas. The dominance of vertical and horizontal lines in visual processing can leave the impression that (c) has 4-fold rather than 8-fold symmetry.

In Figure 16(d) the internal cell structure of motif (c) has been simplified by erasing the innermost line segments. The remaining lines are still collinear and so have good continuation. Like (a), this star has two components, although this is not so apparent as their shape is less familiar than a square. This motif also has a higher-order structure as a group of kites arranged around a point. The kite is another Islamic motif - stars with the same outline as the $\{8 / 3\}$ motif were used in Roman ornament but with the rhombic subdivision shown in (e).

\subsection{Cut-and-paste construction}

Figure 17 illustrates the construction of a star we shall use later. It is unusual in that it is made by cutting up two symmetrical stars and re-assembling some of the pieces. Figure 17(a) is the outline of the 8-pointed star from Figure 16(a), and (b) is a 10-pointed star that also has $90^{\circ}$ corners where it meets its circumcircle. In (c) two sectors from (a) are interleaved with two sectors from (b) to make a 9-pointed star. The result is shown in (d) without its construction lines; it is noticeable that its spikes are uneven and vary in size.

\subsection{Hankin's construction}

Figure 18 shows a selection of shapes constructed by applying Hankin's method to some 7-sided convex polygons: the tip of a spike is located at the midpoint of an edge of the polygon, and lines with an incidence angle of $671 / 2^{\circ}$ to the edge are grown into the interior of the polygon until they meet one another. The circle and radial tics are not part of the construction, but are included to provide a common frame of reference, enabling comparison between the figures. The corners of the polygon and the choice of incidence angle are the parameters that control the construction. These examples show that the shape of the resulting 'star' is quite sensitive to small perturbations in the polygon. In fact, the resulting shape can sometimes become degenerate and cross itself.

Various constraints have been placed on the control polygon.

- In (a) the polygon is a regular heptagon and the resulting star has 7-fold symmetry. The corners of the polygon lie where the radial tics intersect the circumcircle.

- In (b-d) the polygon is equilateral but the corners are otherwise free. 


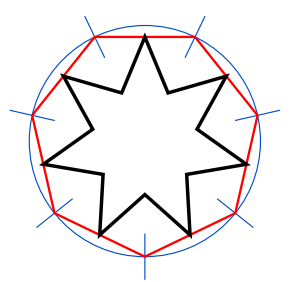

(a)

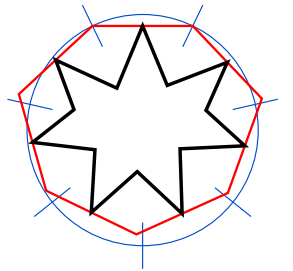

(b)

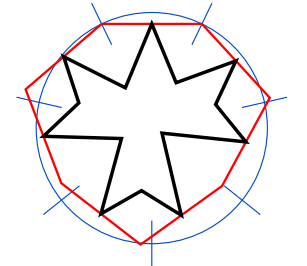

(c)

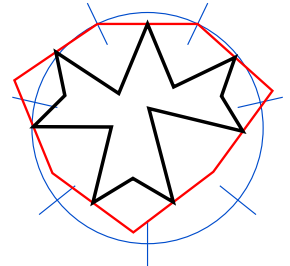

(d)

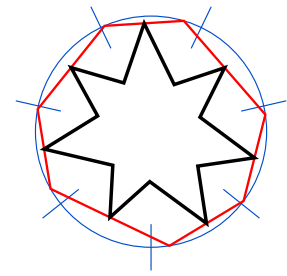

(e)

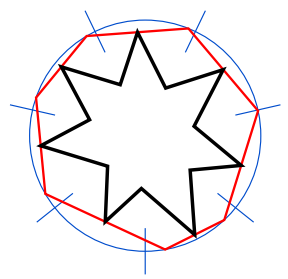

(f)

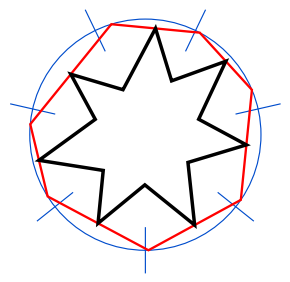

(g)

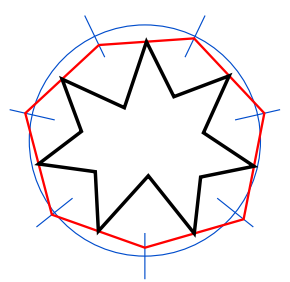

(h)

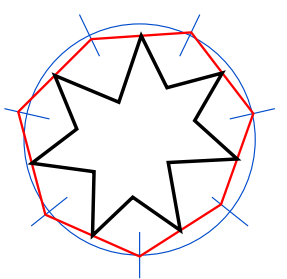

(i)

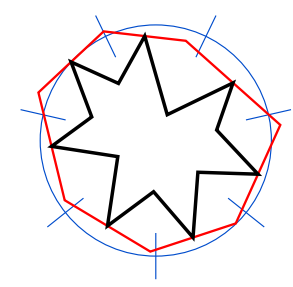

(j)

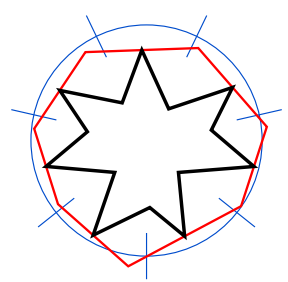

(k)

Figure 18: Spiky shapes constructed from 7-sided polygons using Hankin's method. 


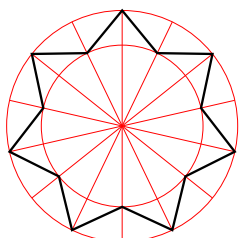

(a)

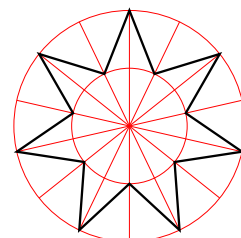

(b)

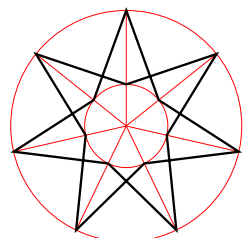

(c)

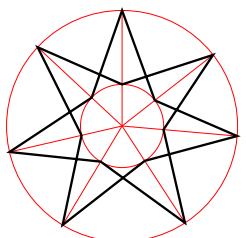

(d)

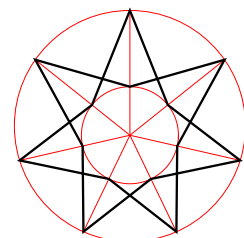

(e)

Figure 19: Making stars with the wheel construction.

- In (e-g) the corners of the polygon are constrained to lie on the circle and have a small random displacement away from the tics.

- In (h-i) the corners of the polygon are constrained to lie on the radial tics and have a small random displacement away from the circle.

- In (j-k) the corners are unconstrained and have a small random displacement from those of the regular polygon.

Although the corners of the control polygon are constrained in these examples and do not move far, the perturbations seem to become amplified and have a large effect on the apparent regularity of the 'star'. The resulting shapes are zig-zags that close up, but they seem less star-like than some of the other examples, and are more reminiscent of the spiky caption bubbles in comics used for explosive sounds or shouting. Placed among this crowd of distractors, even the 7-fold star (a) struggles to look special.

\subsection{Wheel construction}

One problem with Hankin's method is that, while the control polygon does dictate the shape of the star, the control parameters (corners of the polygon) act through intermediate points and are only indirectly related to visual properties of the resulting star. The construction described in this section models the idea that a star is a zig-zag that closes around on itself, using scaffolding whose control parameters are directly related to features of the star.

A wheel is a framework consisting of two circles, one inside the other, and a set of spokes radiating out from a focal point to meet the outer circle. The star is constructed by drawing a line between adjacent spokes that bounces alternately off the inner and outer circles. Figure 19(a) shows a simple example.

The method is very flexible and the other parts of Figure 19 illustrate the consequences of varying some of the parameters.

- Reducing the radius of the inner circle produces a star with sharper spikes - see (b).

- In (a) and (b) the spokes come in two kinds depending on whether they define the inner or outer corners of the star. In fact, all the spokes can serve both roles, as shown in (c). In this case, the method automatically produces the characteristic ring of kites found in Islamic star motifs.

- In (d) the spokes are not evenly spaced. This kind of adjustment enables the artist to control the alignment between motifs. As we shall see later, this becomes important when combining stars of different types in a design. 


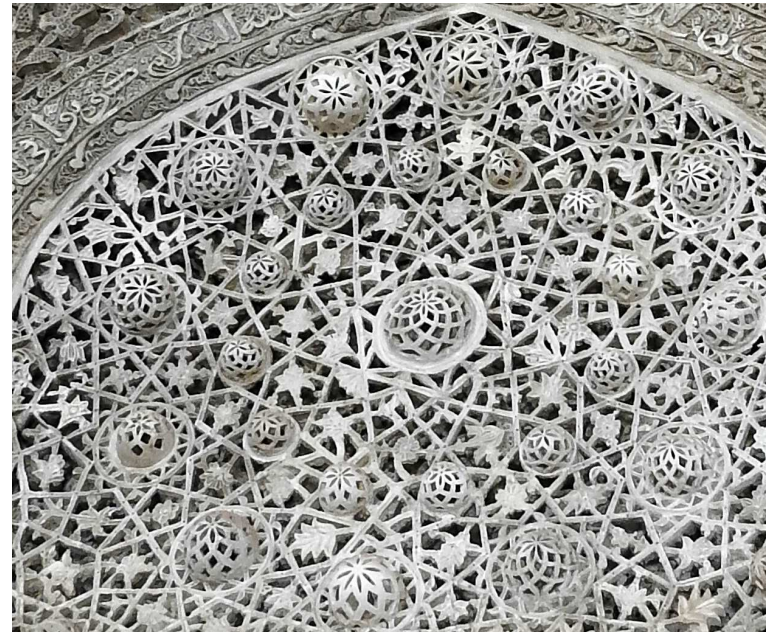

(a)

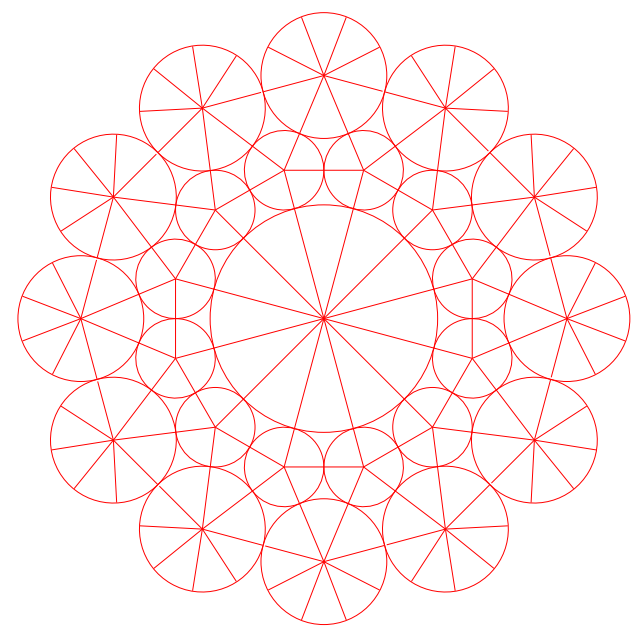

(b)

Figure 20: Part of the thirteenth century mihrab in the Friday Mosque, Urmia, Iran.

- In (e) the circles are not concentric and the inner circle is shifted down. The spokes are equally spaced and radiate from the centre of the inner circle. This device produces stars with larger spikes at the top and smaller, closer spikes at the bottom, and facilitates a change of scale in a design.

Figure 20 illustrates how wheels can be used in practice. Part (a) shows a carved mihrab that contains irregular 5-pointed and 8-pointed stars, and (b) shows a packing of tangent circles that underlies its composition. The circles determine the layout and proportions of the stars - they are the outer circles of the wheels. Connecting their centres as shown produces spokes of the wheels that are irregularly spaced within a wheel but well aligned with neighbouring wheels. Choosing inner circles for the wheels determines the endpoints of the line segments that form the pattern, and the spikiness of the resulting stars. In this case, ornamental hemispherical domes have been added to the design, which sit on the incircles of the stars.

The wheel construction is robust: the control parameters can take widely varying values, yet the results still look star-like. The geometry of the wheel seems to embody the essential characteristics and constrains the shape within visually recognisable limits. This method is versatile and includes the family of Classical stars, but some stars cannot be created in this way (for example, the 9-pointed star in Figure 17(c) does not have an incircle).

\subsection{Modelling regularity}

To ask the question 'what makes a good star?' suggests that we have a well-defined concept of star, that we can recognise when a shape belongs to the category of stars, and that shapes can be starlike to varying degrees so that some shapes are better stars than others. Performing classification of this kind requires some internal representation or encoding of

\footnotetext{
${ }^{11}$ The photograph is cropped from an original produced by user Lombardia, which is available at https://commons.wikimedia.org/wiki/File:The_Mihrab_of_Jameh_Mosque_of_Urmia-1.jpg under a creative commons attribution licence.
} 
shapes, and cognitive processing to enable object recognition - topics we shall consider in part II [16]. We shall presume here that the candidate shapes are starlike; what we are asking is which of them have good form.

Good form (also called good Gestalt or figural goodness) has no agreed definition or metric, but refers to the strength of the perceptual response to structure in the input data. Experiments show that some shapes or configurations elicit faster response times or are consistently preferred to others. As noted in $\S 4$, the fluency hypothesis asserts that these factors are linked: successful processing has a small reward and people derive pleasure from structures that are easily processed. Perceptual fluency provides the key element in our definition of order or regularity in a pattern: regularities are structures or features of the data that are processed fluently. Thus, regularity depends on properties of the pattern and properties of the visual system.

The relationship between input and processing is crucial. Our visual system evolved to process images of our natural environment, and structures for which we have developed specialised processing have survival value. Ultimately, our low-level feature extractors exist because they contribute to the detection and recognition of danger, food, mates, and so on. Of the natural structures that we listed in $\S 3$, the one best suited to modelling stars is growth from a point source. With the exception of Figure 18(d), the examples of stars we have constructed are all star-shaped in the mathematical sense (they have an interior point from where you can view the entire boundary contour). A growth-point detector might respond to nested closed curves (like tree rings), or lines that converge towards a common point. ${ }^{12}$ There is some evidence that non-experts (who are less influenced by cognitive considerations such as style or taste) find average stimuli more pleasing than novel ones. This suggests that the average case becomes the exemplar modelled in a low-level feature detector. The discriminating characteristics distilled into our hypothetical growth-point detector would consist of concentric circles and radial lines. The strength of its response depends on how closely the elements of the input match this template of an ideal form. We expect man-made objects with perfect point symmetry would be super stimuli, matching the exemplar more closely than naturally occurring objects, and generating a larger response.

Let us consider how such a growth-point detector would match our candidate stars.

- The Classical star motifs in Figure 16 have a nested structure with a central cell surrounded by one or more rings of other cells. The nesting is ideal: equivalent vertices lie on a set of concentric circles. The outer cells also provide a radial feature.

- The zig-zag nature of the simple closed curve in Figure 17(d) defines a nested structure as the outer and inner corners fall naturally into two levels. The outer ones lie on a circle, but the inner ones do not.

- The stars in Figure 18 are also zig-zag polygons, but here a division into nested levels is not so clearly defined. The outer vertices always lie on a convex polygon, but the inner ones are less well organised - connecting them in sequence does not always form a convex shape.

In some cases we can find radial structure. When the control polygon has a circumcircle, as in Figure 18(e-g), the bisectors of the spikes meet at the centre of the circle.

\footnotetext{
${ }^{12}$ Such a mechanism is described in [45].
} 


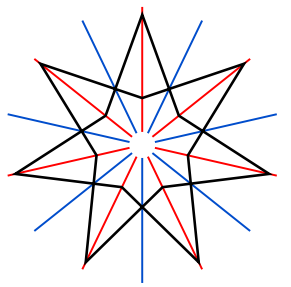

(a)

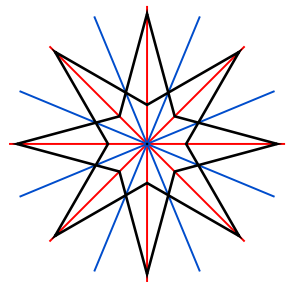

(b)

Figure 21: Local mirror lines are situated differently in odd and even stars.

In other examples the bisectors do not converge and it is difficult to locate a focal point.

- The wheel framework used in Figure 19 correlates with the growth-point template very closely: the inner and outer circles are nested and the spokes lead to radial features.

Matching the growth-point detector is not the only source of rewards. Stars have other salient features such as mirror lines.

Consider the symmetry properties of a motif with $n$-fold symmetry, and take as examples the stars in Figure 21. They have 7-fold and 8-fold symmetry, but what matters is that they illustrate odd and even values of $n$. The coloured lines indicate local mirror lines: the red ones capture the bilateral symmetry of a kite, and the blue ones capture the relationship between a pair of adjacent kites. Pairs of local mirrors on opposite sides of the star are aligned and combine to form a global mirror line of the whole motif. However, when $n$ is odd, the global mirror is formed of a red and a blue part, while when $n$ is even the global mirrors are monochromatic — both ends red or both ends blue. ${ }^{13}$ Although there are always $n$ equally spaced global mirror lines, the arrangements fall into two distinct families.

Now, consider this as a hierarchical perceptual grouping task: first detect features indicating regularity (local mirrors), then group those features with common attributes. When $n$ is even, collinear local mirrors on opposite sides are of the same kind and can be grouped to form a larger, stronger feature. However, when $n$ is odd, opposing local mirrors have different attributes and are not grouped. If this model of perception is valid, stars with even $n$ should provoke stronger responses than stars with odd $n$.

A lot of experimental work has been done on human responses to symmetry, but very little of it addresses this question. Some support comes from Wenderoth and Welsh [67], who studied responses to patterns with $n$ mirror lines for $1 \leqslant n \leqslant 4$. These values of $n$ are too small for stars. However, within this range, increasing $n$ gave increased accuracy and reaction time for $n \in\{1,2,4\}$, but performance on 3 -fold patterns was no better than on 2 -fold. This shows that goodness is not a simple monotonic function of $n$.

How are the local mirrors in our candidate stars arranged? The Classical stars $\{n / d\}$ have perfect $n$-fold symmetry, and have all the mirrors illustrated by the examples in Figure 21. The joins in the composite star in Figure 17(c) are not local mirrors; the vertical axis is its only global mirror line. For the stars in Figure 18, each of the sides and angles of the control polygon can be associated with a local mirror: the perpendicular bisectors of

\footnotetext{
${ }^{13}$ This is a structural difference that is independent of the motif and which can be expressed algebraically. In the dihedral point group $D_{n}$ generated by reflections, the elements form a single conjugacy class when $n$ is odd (red-blue lines), and two classes when $n$ is even (red lines and blue lines).
} 


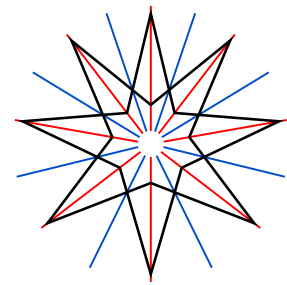

(a)

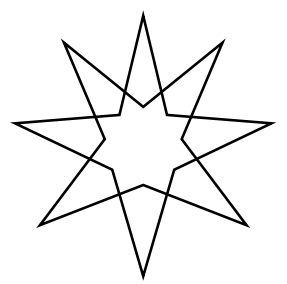

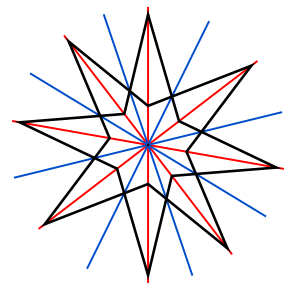

(b)

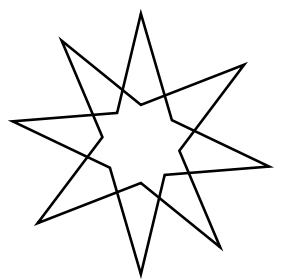

Figure 22: Local mirror lines in even stars do not function as global mirrors when they are perturbed.

the sides can be red mirror lines, and the angle bisectors can be blue mirror lines. However, the sides of the star that would be mirror images under these reflections are often different lengths, so many of the bisectors do not function as local mirrors.

Stars generated by the wheel framework are more predictable. When the wheel has concentric circles and equally spaced spokes, the star has perfect symmetry. Uneven spacing of the spokes or an off-centre hub produces an irregular star, but the local mirrors are still well defined. For a star with an odd number of points, the local mirrors are independent and can be displaced with little impact on the goodness. The situation is more complicated for a star with an even number of points. Figure 22 shows two irregular 8-pointed stars, both with and without annotations marking the local mirror lines. The left halves of (a) and (b) are the same; (a) is completed by reflection in the vertical mirror, and (b) is completed by 2 -fold rotation so that the opposing local mirrors are aligned. In (a) the local mirrors are independent, just as they are in odd stars. However, in (b) there is a conflict because, although alignment and colour of the local mirrors are matched, the grouped lines do not function as global mirrors.

In summary, the model allows us to make the following predictions.

- The zig-zag stars produced by the wheel construction are better than those produced by Hankin's algorithm.

- Stars that are a ring of kites are better than zig-zag stars. This may be because the additional structure makes the local mirror lines easier to locate, or because the grouping of similar forms into a ring also generates a reward.

- Symmetric stars with an even number of points are better than those with an odd number of points.

- If grouping two orthogonal global mirror lines of the same colour into a cross is a salient feature then $4 n$-fold symmetry is stronger than $(4 n+2)$-fold symmetry so that stars with 8 or 12 points are better than those with 6 or 10 points.

- Slightly irregular spacing is less noticeable with odd stars than with even stars.

\section{Arrangements of stars}

So far we have studied various aspects of patterns as individual properties. Let us now consider how an artist creates a composition by bringing motifs together. Artists do not have a completely free choice over the selection and placement of motifs. While aesthetic 
judgement is required, stylistic rules and design principles, and the laws of geometry place some constraints on the relationships.

First let us consider the interaction between local and global symmetries. If the centre of an $n$-fold motif is placed on a $p$-fold rotation centre of the pattern then $n$ must be a multiple of $p$. Patterns produced by repeating a template are periodic, and the Crystallographic Restriction forces $p$ to be $2,3,4$ or 6 . Hence $n$ must be a multiple of 2 or 3 . Furthermore, of the five symmetry types that occur when replicating rectangular templates by reflection, only $p 6 m$ has 3 -fold rotation centres. In all the other cases, stars placed on rotation centres must have an even number of points.

There is no a priori reason why the local and global rotation centres should coincide. Indeed, we shall see below examples where they do not. However, this does not occur in traditional patterns unless separation of the two kinds is forced because the number of points in the star is not a multiple of 2 or 3 . This suggests that placing stars on rotation centres is one of the design principles, either explicitly or as the consequence of some other principle such as 'place the star in a corner of the template'. This rule simplifies the design process considerably as the possible locations of star centres are given.

The artist still has to establish connections between the motifs, which includes setting alignment. The different options can be described using the notation of Figure 21: the local mirrors of neighbouring stars can be aligned homologously (red-red or blue-blue), or mixed (red-blue) as with the 10-pointed stars in Figure 2.

Now we have reviewed the rules on arranging symmetric motifs, the problem of selecting suitable combinations of stars remains. Using two or three different primary stars in a composition introduces further geometric constraints as the motifs must be compatible with each other to facilitate the desired alignment of local mirrors. Artists developed an empirical understanding of the geometric restrictions hundreds of years before mathematicians became interested in repeating patterns and established the underlying theory. Some of them also seem to have delighted in the challenge of circumventing these inherent limitations of the method. The following examples show how symmetry can be broken so long as other visually salient cues are preserved.

If we judge goodness by the number of occurrences then the pattern shown in Figure 23 is one of the most successful Islamic star patterns. It can be found in many places across the Islamic world from Central Asia to Spain with examples dating from the twelfth century onwards. From the foregoing discussion, it seems likely that the following properties contribute to its attraction:

- All the crossings are transverse 4-valent vertices - there are no T-junctions or deviations.

- The large and small star motifs are derived from the Classical forms $\{10 / 4\}$ and $\{5 / 2\}$, respectively. They have 10 -fold and 5 -fold symmetry and so are forbidden as global rotation centres (the 10-pointed star is placed on a global 2-fold centre).

- The stars are properly aligned point-to-point and mesh together naturally.

- The blue rectangle marks a template replicated by reflections in its edges. It has a 2 -fold gyration point (a rotation centre not on a mirror).

- One of its Hankin diagrams is overlaid in red on the left of the figure. Although it is unlikely to be involved directly in visual processing, this segmentation reveals a 


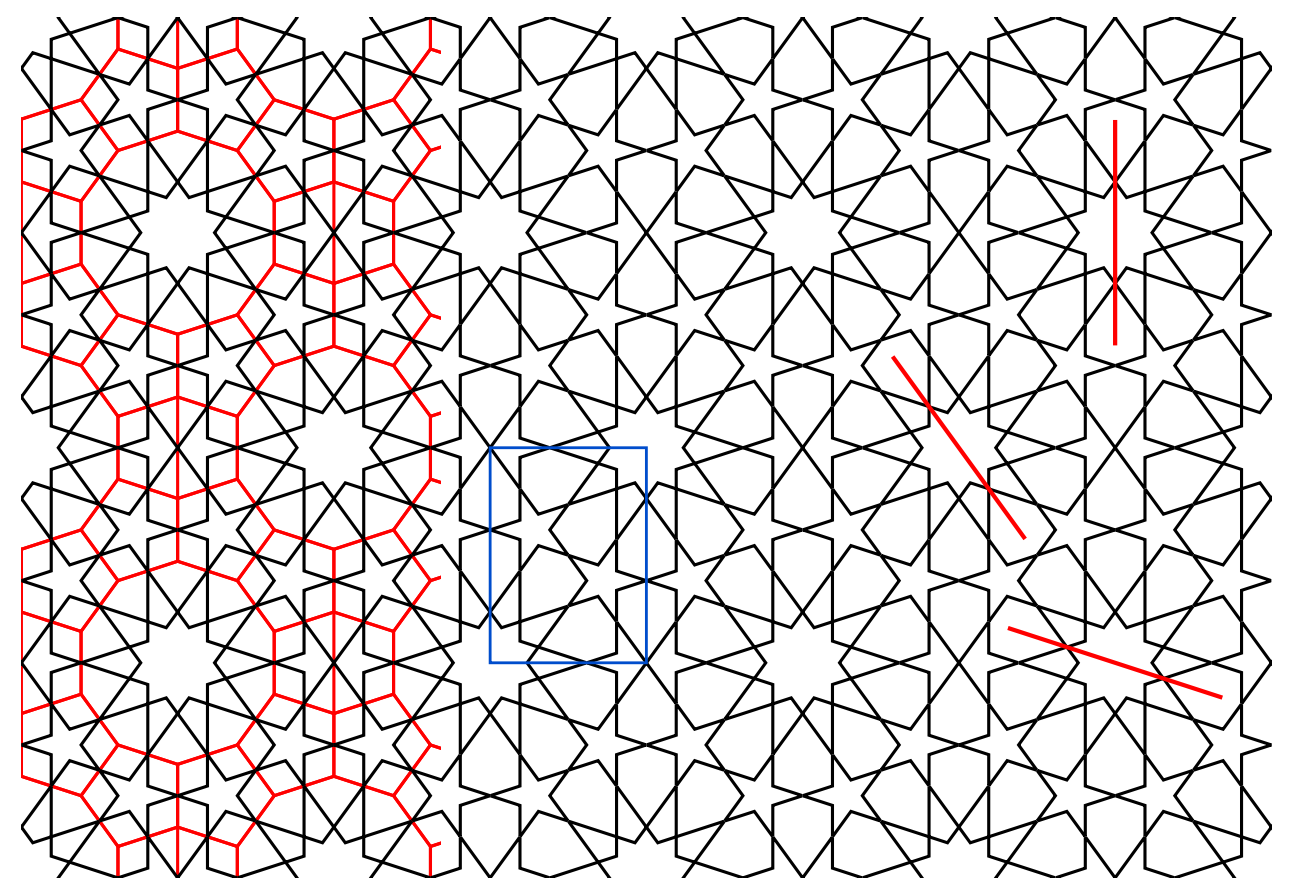

Figure 23: One of the most common and widespread star patterns.

simple underlying structure: all the polygons are convex, the decagon and pentagon are regular polygons, and all the edges meet the pattern at their midpoint.

- The lines within the pattern run in only five directions: vertical plus two mirror-image oblique pairs. A line from each family is marked in red on the right of the figure.

- Interpolating across the gaps between the vertical line segments produces parallel lines running uninterrupted down the panel.

- The family of line segments at $36^{\circ}$ from vertical can also be interpolated to produce oblique lines running uninterrupted across the panel. Those which pass through the arrow motifs form a diaper pattern or diamond lattice.

- The bilateral convex hexagon compartments that sit between the arrow-shaped compartments are congruent to those between the kites in the star motifs. Thus, the same shape appears in different contexts.

Notice that the pattern has different structural properties in each of the cardinal directions. Whichever family of parallel interpolated lines we choose, the intervals between the lines come in two sizes that we shall call fat and thin. In the family of vertical lines the fat and thin intervals alternate as we scan across the pattern. For the family of lines at $36^{\circ}$ from vertical, the repeating sequence of intervals is fat-fat-thin. The family of line segments at $72^{\circ}$ from vertical form short runs of interpolated segments, but these are interrupted where the fat and thin channels switch position. This variety makes the pattern less predictable and so may also add to its interest. 


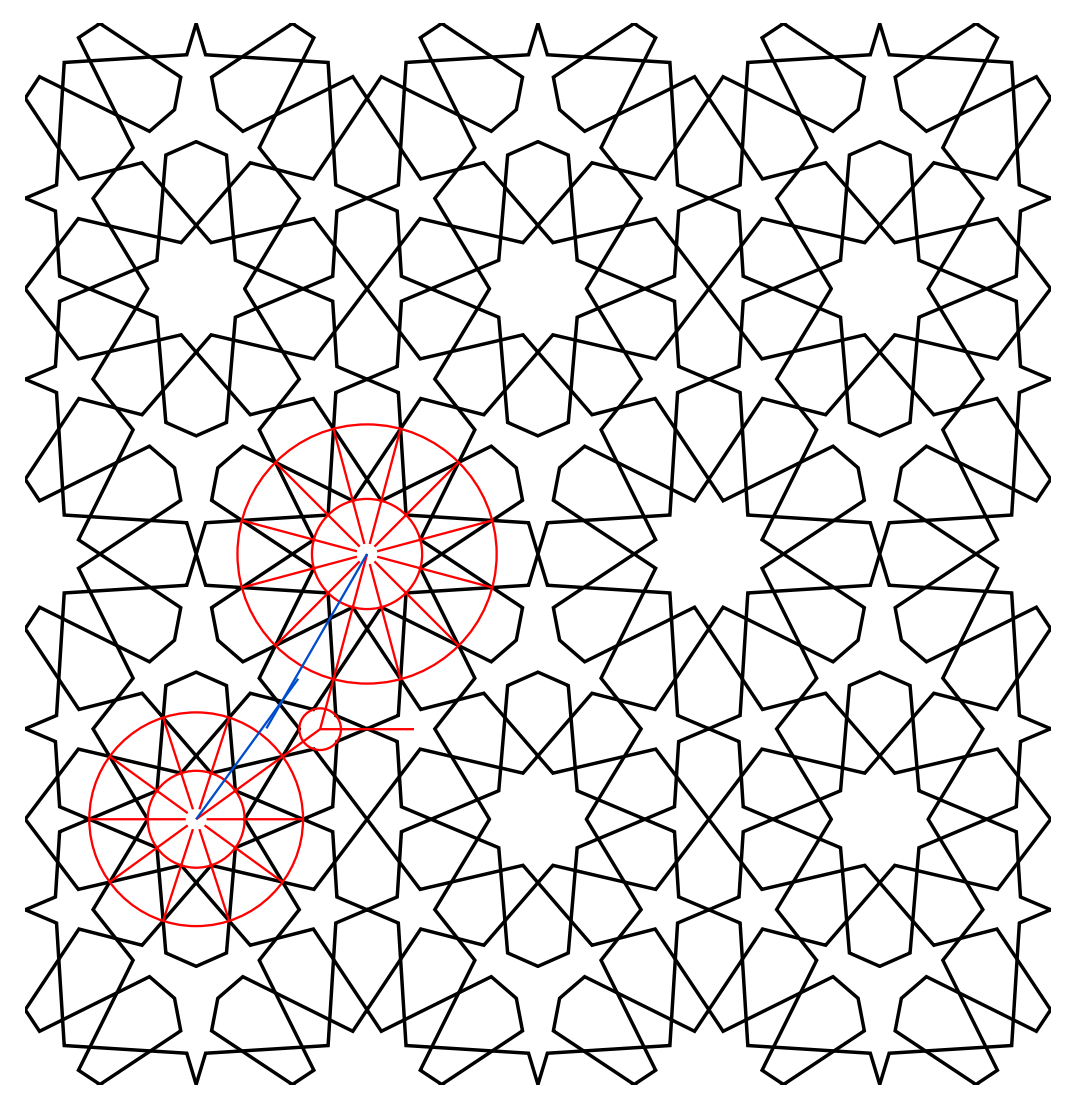

Figure 24: Arrangement of 10-pointed and 12-pointed stars. The primary stars are symmetrical but some local mirrors (blue) are misaligned. Developed from panel 44 of the Topkapr Scroll. 


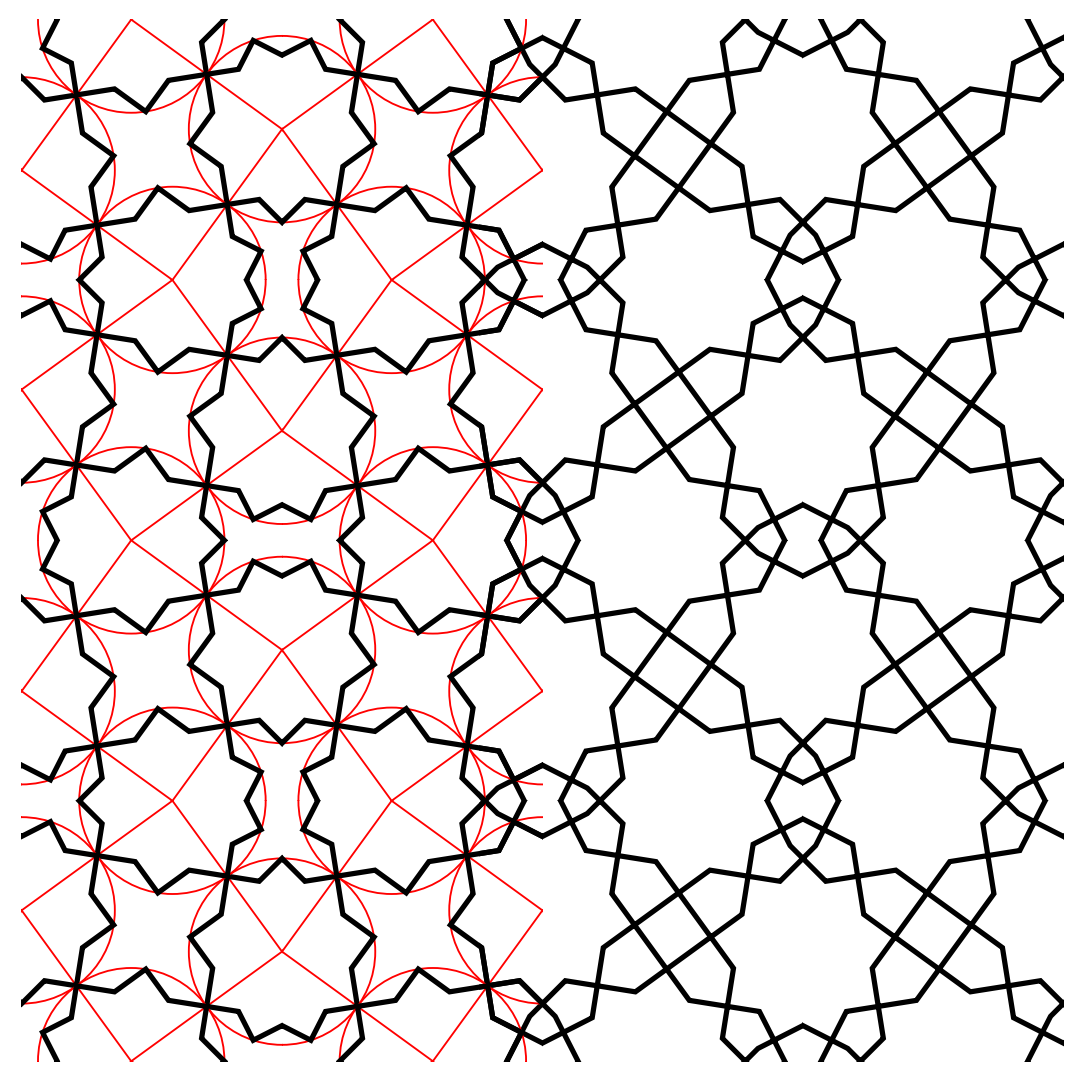

Figure 25: Arrangement of irregular 9-pointed stars with good alignment. From the Seljuk Mosque (1105) in Barsian, Iran.

Let us now examine some examples where the parts are not so well coordinated. Figure 24 is a pattern developed from the template in panel 44 of the Topkapi Scroll, and shows the results of an experiment to combine 10-pointed and 12-pointed stars. As in Figure 23, the centres of the primary stars are situated at opposite corners of the rectangular template. However, in this case, the stars are of different kinds so the centre of the template is not a gyration point. The primary stars are symmetrical and are made using the wheel construction with equally spaced spokes. The 5-pointed stars have an incircle and, while they do not have 5-fold symmetry, the alignment of a 5-pointed star with all its neighbouring stars is correct. The most obvious flaw in the pattern is that the local mirrors of the 10-pointed and 12-pointed stars are misaligned, as shown in blue in the figure.

Whereas symmetrical stars are commodity elements in Islamic design that can be assembled in multiple ways, irregular stars are always custom-made in response to a particular problem. Figure 25 shows the pattern that led to the 9-pointed stars constructed in $\S 7.2$; the red lines on the left of the figure are the same as those in Figure 17(c) and reveal how the stars are packed together. On the right of the figure the pattern has been completed by extending the lines forming the stars until they meet each other. The fact that this process creates cross-like motifs with 4-fold symmetry in the red squares is not an accident - the pattern is probably a variant of the common Star and Cross pattern shown in blue in 


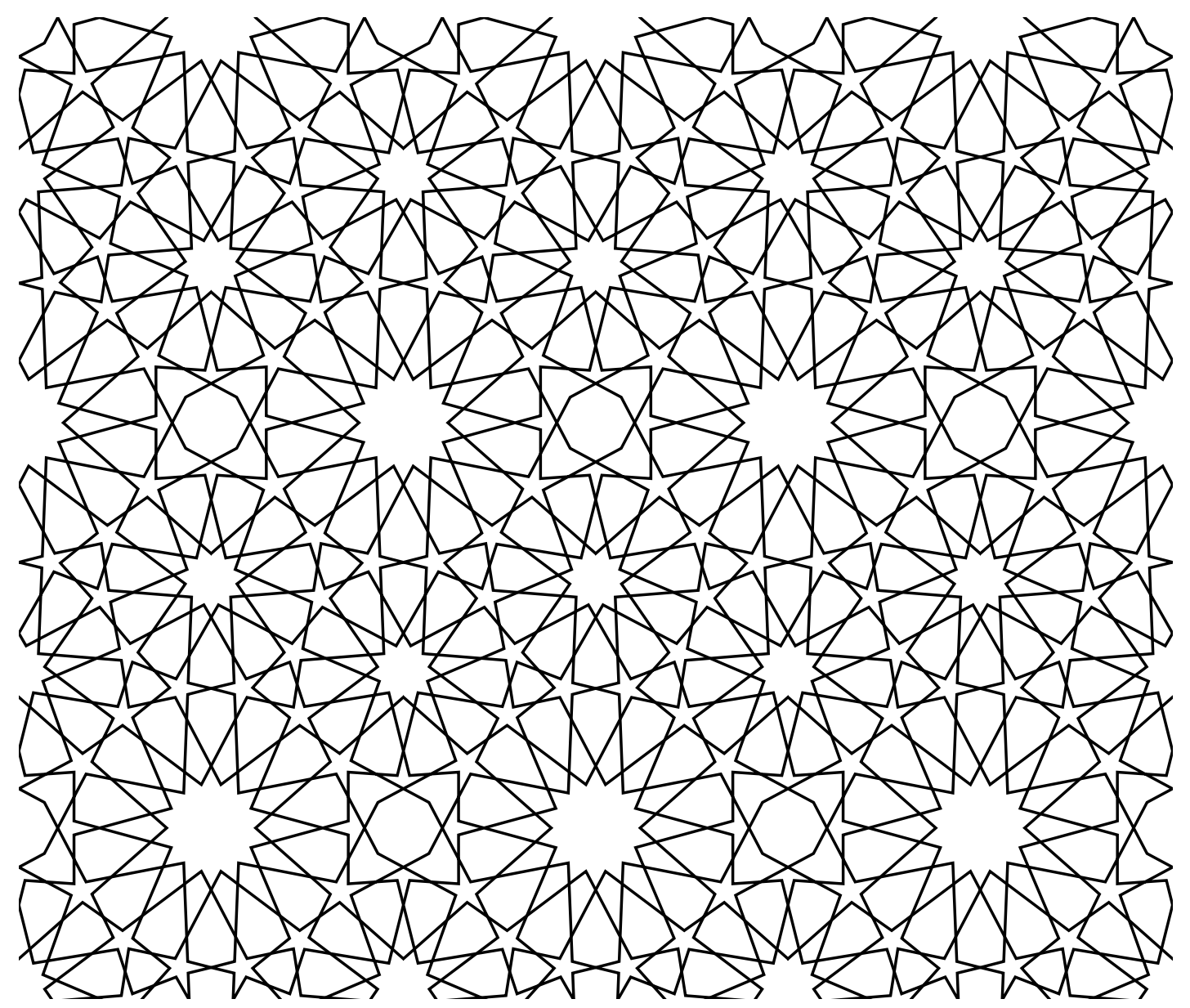

Figure 26: Arrangement of irregular 11-pointed and symmetrical 14-pointed stars with good alignment. Reconstructed from archaeological remains of the Mudhafaria Minaret (1190), Erbil, Iraq.

Figure 11(b). The cross motif is retained and the stars are adapted to fit the new context. However, the resulting 9-pointed stars seem rather clumsy. The noticeable variation in tooth size, combined with the fact that the stars occur in four different orientations within the pattern, makes it difficult to compare the motifs, and it is not apparent that the stars are all congruent.

Figure 26 shows a more subtle and discerning application of irregular stars. The symmetrical 14-pointed stars are centred on opposite corners of the template, while the 11-pointed stars have only bilateral symmetry and are centred on the edges of the template. These primary stars and the secondary 5-pointed stars are all made with the wheel construction, and all local mirrors are correctly aligned. This is one of a small group of illicit patterns constructed in the Seljuk period in which motifs incompatible with each other and with the standard lattices of repeating patterns are combined with no indication that anything out of the ordinary has occurred. Some distortion or irregularity must be present, but it is skillfully disguised by preserving the fundamental features that are important for visual perception and interpretation. It is not until you try to reconstruct the pattern that you 
discover genuinely symmetrical parts do not fit together as shown.

We remarked near the end of $\S 6.1$ that the rectangular form of the $p 6 m$ template resembles a $\mathrm{cmm}$ template. This is evident when comparing Figures 5 and 26. Both patterns combine two primary stars: the large ones (12 or 14 points) are arranged in a horizontal rows across the panel; medium ones (9 or 11 points) are in zig-zag rows in between. Both templates have large even stars in opposite corners, medium-sized odd stars on the vertical edges, and a 2 -fold rotation point in the centre of the rectangle. Figure 5 has additional global symmetries, but the piece shown is not large enough to appreciate this, and this fact may also help to make it appear less rigid and predictable.

Finally, let us return to the composition of Figure 1. The primary motifs used in this pattern are rings of kites like the one shown in Figure 27(a). The large rings have 17 kites and the smaller rings have 13 kites.

The pattern is produced by reflections from a rectangular template with a gyration point in the centre (symmetry type $\mathrm{cmm}$ ). Since 13 and 17 are both odd, the primary motifs cannot be placed at the corners of the template. Moreover, 13 and 17 are prime (hence not multiples of 2 or 3 ) so they cannot be centred on other global rotation points of the pattern either. The geometries of symmetric 13 -fold and 17-fold motifs are not compatible so some adjustments must be made to facilitate the alignment of local mirror lines.

In Figure 27(a) overlapping congruent kites are equally spaced around the centre. Varying the spacing by a small amount, as illustrated in (b), produces noticeable differences in the size of the overlaps, and this irregularity upsets the balance of the motif. In (c) the spacing of the kites is the same as in (b), but the kites are no longer congruent to each other: the height of the kites remains the same but their width has been adjusted so that the overlaps have the equal width. In this way we can create irregular motifs to control the alignment in the pattern without drawing attention to the deception.

In Figure 28 the red lines mark four copies of the rectangular template and all the alignments that have been enforced between neighbouring stars. To complete the pattern, the kites are interlaced. Mathematically, this destroys all the mirror symmetry, but visually the axes are still salient features.

\section{Discussion}

We have explored the visual properties of Islamic star patterns to identify features that contribute to a positive response in the viewer. We have also tried to work out whether such features are a consequence of the practical processes of pattern production, the laws of geometry, or are under the control of the artist.

In this paper we have focussed on low-level perceptual processing. We have taken an ecological approach, meaning that our visual system is adapted to process images of the natural environment in which it evolved, structures in the data for which we have developed specialised processing have survival value, and processing is based on statistical properties of the environment that have either become innate or are learned empirically very early in life by exposure to visual data. Such processing is direct, not mediated by representations or knowledge (we shall explore these more cognitive processes in part II [16]). This approach

provides a key element in our perceptual model of a star. Flowers are an example of a common natural structure caused by growth from a central point. Detectors for such features may respond to nested rings or radial lines, and artificial stimuli such as stars will 


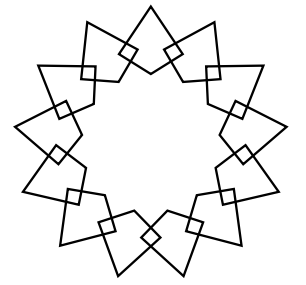

(a)

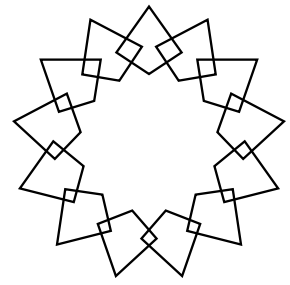

(b)

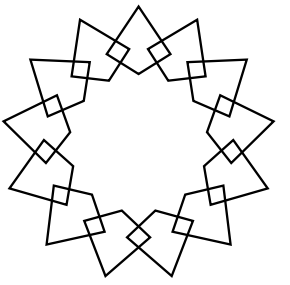

(c)

Figure 27: Similar overlaps disguise unequal spacing.

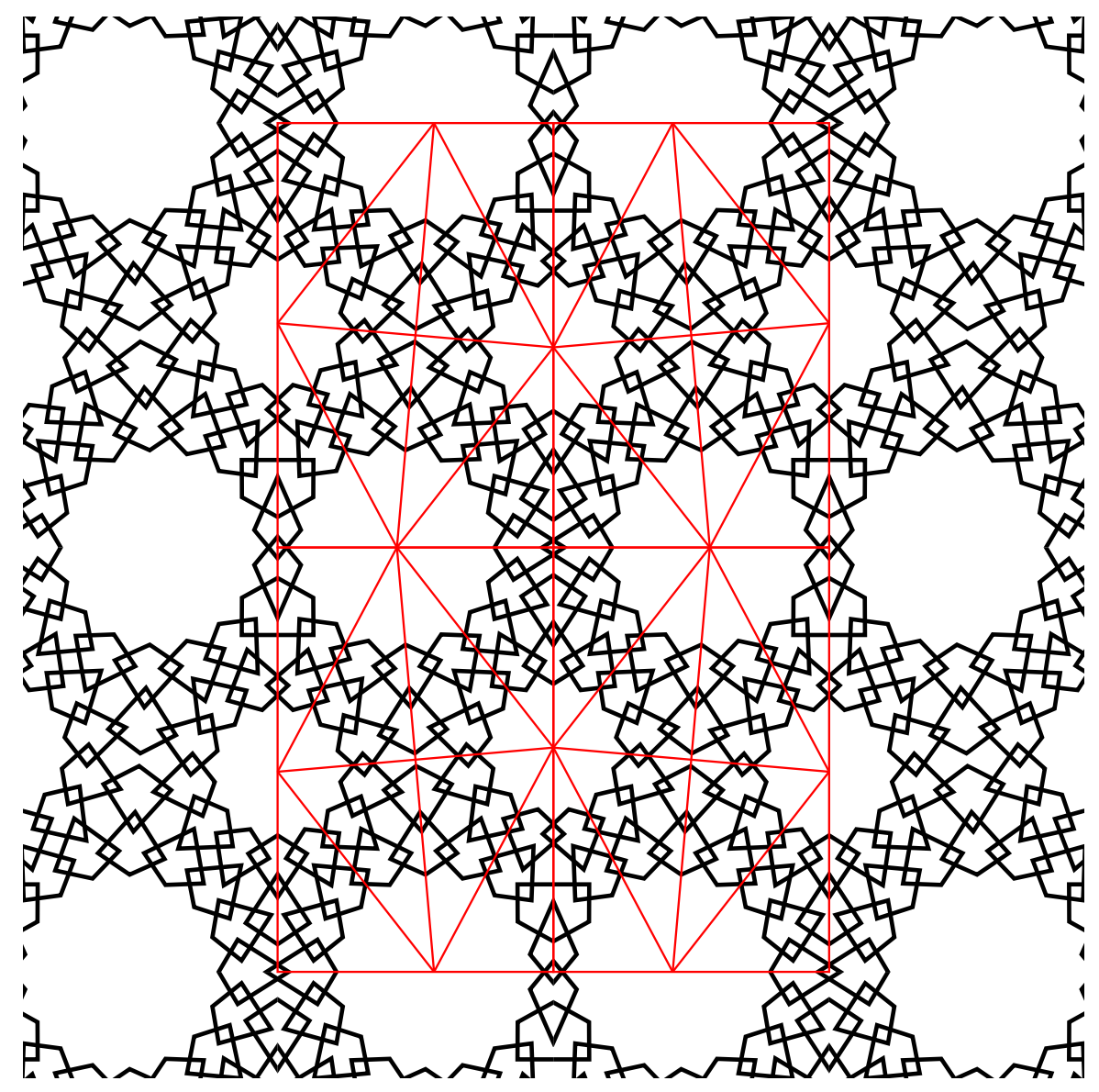

Figure 28: Construction of Figure 1. 
also trigger them.

Structures in visual data for which we have evolved fast specialised processing are said to be processed fluently. We defined a regularity in the data to be a fluently processed feature, and noted that experimentally determined regularities seem to coincide with non-accidental relationships in the data. We have also adopted the perceptual fluency hypothesis, which asserts that successful processing generates a reward. Thus the pleasure derived from a star motif can be accumulated from several features, each of which gives a small reward: a central point detection, local mirrors within or between neighbouring spikes of the star, and the grouping of local mirrors into larger global mirrors. This led us to predict that even stars are better than odd stars, but odd stars do not need to be so accurately drawn.

In our survey of psychophysical properties we noted that the geometry of perceptual space is far more attuned to affine properties of the data (straightness, collinearity, parallelism, convexity) than it is to metric properties (lengths and angles). This means that maintaining alignment of key elements in a pattern, even imaginary elements such as mirror lines, is more important than high accuracy in the division of an angle into equal parts.

Mirror symmetry is a dominant feature in Islamic geometric patterns, but it is unclear whether this is a consequence of the practicalities of pattern production or stylistic conventions, rather than psychological processes. Virtually all traditional Islamic star patterns can be produced from a template that is repeated by reflections in its sides. The fact that most patterns are applied to decorate a rectangular panel, and the convention that the panel should be covered by a whole number of templates, means that the templates are also rectangular. Together, these two observations imply that that configurations of symmetry elements in star patterns conform to one of five symmetry types: $p m m, p 4 m, p 6 m, c m m$, or $p 4 g$. The last two types have gyration points (rotation centres that are independent of the mirrors).

The symmetry type of a pattern determines the location and kind of its global rotation centres. Large primary stars provide easily detectable local centres of symmetry. There is no reason why the local centres and global centres in a pattern should coincide. When combined together they reinforce each other, producing a strong focal point, whereas separating them creates tension as the two classes compete for attention. In traditional star patterns, stars are situated on compatible ${ }^{14}$ global centres whenever possible. It is unclear whether this is to avoid creating tension in a pattern, or just because it is easier to allow the laws of geometry to dictate the location and distribution of the stars by using the predetermined global centres to position them. Some stars ${ }^{15}$ are not compatible with any global centres, but still appear in patterns, so avoiding tension in a pattern is not a key goal for an artist.

Besides using stars that are incompatible with the underlying symmetry types, medieval artists also created patterns containing combinations of stars whose geometries are incompatible with each other. In these cases, it is not possible to have perfectly symmetric stars and for the stars to be properly aligned with their neighbours. Either the stars are imperfect or the alignment is out or both. Our model of star goodness predicts that, in order to achieve the best results, stars with an even number of points should be symmetrical, and that irregularly spaced spikes in a star with an odd number of points will not be noticeable. We have seen examples that apply these principles to create illicit patterns.

Finally, we noted that artists will exploit and enhance serendipitous emergent features

\footnotetext{
${ }^{14} \mathrm{An} n$-fold motif can only be placed on a $p$-fold rotation centre if $n$ is a multiple of $p$.

${ }^{15}$ Any $n$-pointed star where $n$ is not a multiple of 2 or 3 .
} 
created by automatic visual processing, such as illusory curves, when they appear in a pattern.

In part II we shall explore the more puzzle-like aspects of Islamic geometric patterns, and consider how the knowledge and experience of the viewer contributes to the interpretation of the data.

This research received no specific grant from any funding agency in the public, commercial, or not-for-profit sectors.

\section{Figure sources}

Figure 2 is from a photograph by Alan Davey, which is available at https://www.flickr.com/photos/adavey/4957590217 under a creative commons attribution licence.

Figure $8(\mathrm{~b})$ is by the author.

Figure 15 shows photographs MET 006 and IND 0820 from David Wade's archive at http://www.patterninislamicart.com/

which are used with permission.

Figure 20 is cropped from an original photograph by user Lombardia, which is available at https://commons.wikimedia.org/wiki/File:The_Mihrab_of_Jameh_Mosque_of_Urmia-1.jpg under a creative commons attribution licence.

\section{References}

[1] S. J. Abas and A. S. Salman, Symmetries of Islamic Geometrical Patterns, World Scientific, 1995.

[2] M. K. Albert and D. D. Hoffman, 'Genericity in spatial vision', Geometric Representations of Perceptual Phenomena, eds. R. D. Luce, D. D. Hoffman, M. D’Zmura, G. Iverson and A. K. Romney, Psychology Press, 1995, pp. 95-112.

[3] R. Arnheim, Art and Visual Perception: A Psychology of the Creative Eye, Univ. California Press, 1974.

[4] C. Balmelle and R. Prudhomme, Le Décor Géometrique de la Mosaïque Romaine: vol 1 Répertoire Graphique et Descriptif des Compositions Linéaires et Isotropes, Picard, 1985.

[5] M. Bertamini and A. D. J. Makin, 'Brain activity in response to visual symmetry', Symmetry 6 (2014) 975-996.

[6] C. Bode, M. Helmy and M. Bertamini, 'A cross-cultural comparison for preference for symmetry: comparing British and Egyptians non-experts', Psihologija 50 no. 3 (2017) 383-402.

[7] J. Bonner, Islamic Geometric Patterns, Springer, 2017. 
[8] S. R. Broadbent, 'Simulating the ley hunter', J. Royal Statistical Soc. Series A 143 (1980) 109-140.

[9] V. F. Bruce, P. R. Green, M. A. Georgeson, Visual Perception: Physiology, Psychology and Ecology, Psychology Press, Taylor and Francis, 2003.

[10] N. J. Bullot and R. Reber, 'The artful mind meets art history: toward a psychohistorical framework for the science of art appreciation', Behavioral and Brain Sciences 36 (2013) 123-180.

[11] J. H. Conway and D. H. Huson, 'The orbifold notation for two-dimensional groups', Structural Chemistry 13 (2002) 247-257.

[12] M. C. Corballis and C. E. Roldan, 'On the perception of symmetrical and repeated patterns', Perception and Psychophysics 16 (1974) 136-142.

[13] S. Coren and J. S. Girgus, 'Principles of perceptual organization and spatial distortion: the Gestalt illusions', J. Experimental Psychology: Human Perception and Performance 6 no. 3 (1980) 404-412.

[14] P. R. Cromwell, 'Celtic knotwork: mathematical art', Math. Intelligencer 15 no. 1 (1993) 36-47. Reprinted in Mathematical Conversations, eds. R. J. Wilson and J. Gray, Springer Verlag, 2001.

[15] P. R. Cromwell, 'A modular design system based on the Star and Cross pattern', J. Math. and the Arts 6 (2012) 29-42.

[16] P. R. Cromwell, 'Looking at Islamic patterns II: making sense of geometry', Preprint 2021.

[17] P. R. Cromwell, E. Beltrami and M. Rampichini, 'The Borromean rings', Math. Intelligencer 20 no. 1 (1998) 53-62.

[18] J. T. Enns, The Thinking Eye, The Seeing Brain: Explorations in Visual Cognition, W. W. Norton and Co., 2004.

[19] J. Feldman, 'Tuning your priors to the world', Topics in Cognitive Science 5 (2013) $13-34$.

[20] W. T. Freeman, 'The generic viewpoint assumption in a framework for visual perception', Nature 368 (1994) 542-545.

[21] J. Freyd and B. Tversky, 'Force of symmetry in form perception', American J. Psychology 97 (1984) 109-126.

[22] B. M. Gibson, O. F. Lazareva, F. Gosselin, P. G. Schyns and E. A. Wasserman, 'Nonaccidental properties underlie shape recognition in mammalian and nonmammalian vision', Current Biology 17 (2007) 336-340.

[23] E. H. Gombrich, Art and Illusion: A Study in the Psychology of Pictorial Representation, Princeton Univ. Press, 1960. 
[24] E. H. Gombrich, The Sense of Order: A Study in the Psychology of Decorative Art, Cornell Univ. Press, 1979.

[25] I. E. Gordon, Theories of Visual Perception, Psychology Press, Taylor and Francis, 2004.

[26] P. Gros, R. Hartley, R. Mohr and L. Quan, 'How useful is projective geometry?', Computer Vision and Image Understanding 65 (1997) 442-446.

[27] B. Grünbaum, 'What symmetry groups are present in the Alhambra?', Notices American Math. Soc. 53 no. 6 (2006) 1-5.

[28] B. Grünbaum and G. C. Shephard, Tilings and Patterns, W. H. Freeman, 1987.

[29] B. Grünbaum and G. C. Shephard, 'Interlace patterns in Islamic and Moorish art', Leonardo 25 (1992) 331-339. Reprinted in The Visual Mind: Art and Mathematics, ed. M. Emmer, MIT Press, 1993.

[30] E. H. Hankin, 'On some discoveries of the methods of design employed in Mohammedan art', J. Society of Arts 53 (1905) 461-477.

[31] R. F. Hess, A. Hayes, and D. J. Field, 'Contour integration and cortical processing', J. Physiology — Paris 97 (2003) 105-119.

[32] C. Q. Howe and D. Purves, 'Natural-scene geometry predicts the perception of angles and line orientation', Proc. National Academy Sciences 102 no. 4 (2005) 1228-1233.

[33] A. D. Jepson and W. Richards, 'What makes a good feature', Spatial Vision in Humans and Robots, eds. L. Harris and M. Jenkin, Cambridge Univ, Press, 1993, pp. 89-126.

[34] C. S. Kaplan, 'Computer generated Islamic star patterns', Proc. Bridges: Mathematical Connections in Art, Music and Science, (Kansas, 2000), ed. R. Sarhangi, 2000, pp. 105112.

[35] C. S. Kaplan, 'Islamic star patterns from polygons in contact', Graphics Interface 2005, ACM International Conference Proceeding Series 112, 2005, pp. 177-186.

[36] D. G. Kendall and W. G. Kendall, 'Alignments in two-dimensional random sets of points', Advances in Applied Probability 12 (1980) 380-424.

[37] K. Landwehr, 'Visual discrimination of the 17 plane symmetry groups', Symmetry 3 (2011) 207-219.

[38] B. Lindström and H.-O. Zetterström, 'Borromean circles are impossible', American Math. Monthly 98 no. 4 (1991) 340-341.

[39] D. G. Lowe, Perceptual Organization and Visual Recognition, Ph.D. Thesis, Stanford Univ., 1984.

[40] C. Muth and C.-C. Carbon, 'The aesthetic aha: on the pleasure of having insights into Gestalt', Acta Psychologica 144 (2013) 25-30. 
[41] R. Navarro, 'The optical design of the human eye: a critical review', J. Optometry 2 (2009) 3-18.

[42] A. Özdural, Interlocks of Similar or Complementary Figures: Collaboration of Mathematicians and Artisans in the Islamic World, 2003. Manuscript available from Brill Online at https://doi.org/10.6084/m9.figshare.5255416.v1. Edited version in The Arts of Ornamental Geometry, ed. G. Necipoğlu, Supplement to Muqarnas, 13, Brill, 2017.

[43] M. Parker, 'Locations of ancient Woolworths stores follow precise geometrical pattern', Press Release, 5 Jan 2010.

http://web.archive.org/web/20100812112158/http://www. standupmaths. com:80/woolworths/

[44] Z. Pizlo, A. Rosenfeld and I. Weiss, 'The geometry of visual space: about the incompatibility between science and mathematics', Computer Vision and Image Understanding 65 (1997) 425-433.

[45] F. J. A. M. Poirier and H. R. Wilson, 'A biologically plausible model of human radial frequency perception', Vision Research 46 (2006) 2443-2455.

[46] J. R. Pomerantz and M. Kubovy, 'Theoretical approaches to perceptual organization: simplicity and likelihood principles', in Handbook of Perception and Human Performance, Vol. 2. Cognitive Processes and Performance eds. K. R. Boff, L. Kaufman and J. P. Thomas, John Wiley \& Sons, Oxford, 1986, pp. 1-46.

[47] D. Purves, Y. Morgenstern and W. T. Wojtach, 'Perception and reality: why a wholly empirical paradigm is needed to understand vision', Frontiers in Systems Neuroscience 9 (2015) article 156.

[48] D. Purves, W. Wojtach and R. B. Lotto, 'Understanding vision in wholly empirical terms', Proc. National Academy Sciences 108 no. 3 (2011) 15588-15595.

[49] V. S. Ramachandran, 'Sharpening up 'The Science of Art': an interview with Anthony Freeman', J. Conciousness Studies 8 (2001) 9-29.

[50] V. S. Ramachandran and W. Hirstein, 'The science of art: a neurological theory of aesthetic experience', J. Conciousness Studies 6 (1999) 15-31.

[51] R. Reber, N. Schwarz and P. Winkielman, 'Processing fluency and aesthetic pleasure: is beauty in the perceiver's processing experience?', Personality and Social Psychology Review 8 (2004) 364-382.

[52] C. Redies, 'Combining universal beauty and cultural context in a unifying model of visual aesthetic experience', Frontiers in Human Neuroscience 9 (2015) article 218.

[53] R. Sarhangi, S. Jablan and R. Sazdanovic, 'Modularity in medieval Persian mosaics: textual, empirical, analytical, and theoretical considerations', Proc. Bridges: Mathematical Connections in Art, Music and Science, (Kansas, 2004), eds. R. Sarhangi and C. H. Séquin, 2004, pp. 281-292. 
[54] S. Sarkar, 'An introduction to perceptual organization', Proc. IEEE Conf. Integration of Knowledge Intensive Multi-Agent Systems (KIMAS, Boston, 2003), 2003, pp. 330 335 .

[55] A. P. Shimamura, 'Toward a science of aesthetics', Aesthetic Science: Connecting Minds, Brains and Experiences, eds. A. P. Shimamura and S. E. Palmer, Oxford Univ. Press, 2012, pp. 3-28.

[56] D. W. Thompson, On Growth and Form, Cambridge Univ. Press, 1961.

[57] J. T. Todd, S. Oomes, J. J. Koenderink and A. M. L. Kappers, 'The intrinsic geometry of perceptual space: its metrical, affine and projective properties', Proc. Fifteenth Annual Meeting International Soc. Psychophysics, eds. P. R. Killeen and W. R. Uttal, 1999, pp. 169-174.

[58] M. S. Treder, 'Behind the looking-glass: a review on human symmetry perception', Symmetry 2 (2010) 1510-1543.

[59] S. van de Cruys and J. Wagemans, 'Putting reward in art: A tentative prediction error account of visual art', i-Perception 2 (2011) 1035-1062.

[60] J. Wagemans, 'How geometrical is human perception of affine and projective shape equivalence?', Geometry and Topology of Submanifolds, VIII, eds. F. Dillen, B. Komrakov, U. Simon, I. Van de Woestyne and L. Verstraelen, World Scientific, 1996, pp. 351360 .

[61] J. Wagemans, 'Perceptual organization', The Stevens' Handbook of Experimental Psychology and Cognitive Neuroscience, Vol 2 Sensation, Perception and Attention, Fourth Edition, eds. J. T. Wixted and J. Serences, John Wiley \& Sons, 2018.

[62] J. Wagemans, L. van Gool, C. Lamote and D. H. Foster, 'Minimal information to determine affine shape equivalence', J. Experimental Psychology: Human Perception and Performance 26 no. 2 (2000) 443-468.

[63] D. Washburn, 'Symmetry analysis of ceramic design: two tests of the method on Neolithic material from Greece and the Aegean', Structure and Cognition in Art, ed. D. K. Washburn, New Directions in Archaeology, Cambridge Univ. Press, 1983, pp. 138-164.

[64] D. Washburn, 'Style, perception and geometry', Style, Society, and Person: Archaeological and Ethnological Perspectives, eds. C. Carr and J. E. Neitzel, Plenum Press, New York, 1995, pp. 101-122.

[65] R. J. Watt, R. M. Ward and C. Casco, C. 'The detection of deviation from straightness in lines', Vision Research 27 no. 9 (1987) 1659-1678.

[66] P. Wenderoth, 'The salience of vertical symmetry', Perception 23 (1994) 221-236.

[67] P. Wenderoth and S. Welsh, 'Effects of pattern orientation and number of symmetry axes on the detection of mirror symmetry in dot and solid patterns', Perception $\mathbf{2 7}$ (1998) 965-976. 
[68] R. Wenger, 'Visual art, archaeology and Gestalt', Leonardo 30 no. 1 (1997) 35-46.

[69] M. Wertheimer, 'Untersuchungen zur Lehre von der Gestalt II', (Investigations in Gestalt theory II: laws of organisation in perceptual forms), Psycologische Forschung 4 (1923) 301-350.

[70] G. Westphal-Fitch, L. Huber, J. C. Gómez and W. T. Fitch, 'Production and perception rules underlying visual patterns: effects of symmetry and hierarchy', Philosophical Trans. Royal Soc. Series B 367 (2012) 2007-2022.

[71] F. Wilkinson, H. R. Wilson and C. Habak, 'Detection and recognition of radial frequency patterns', Vision Research 38 (1998) 3555-3568.

[72] A. P. Witkin and J. M. Tenebaum, 'What is perceptual organization for?', Proc. Joint Conf. Artificial Intelligence Vol 2, (IJCAI, Karlsruhe, 1983), 1983, pp. 1023-1026.

[73] A. P. Witkin and J. M. Tenebaum, 'On the role of structure in vision', in Human and Machine Vision, eds. J. Beck, B. Hope and A. Rosenfeld, Academic Press, 1983, pp. 481-543.

[74] B. Wood, 'Rethinking the ecology of Islamic geometric ornament', J. Utah Academy Sciences, Arts, and Letters 96 (2019) 41-53.

[75] B. Wood, 'Pleasure and meaning in Islamic art: toward a naturalist framework', $J$. Utah Academy Sciences, Arts, and Letters 97 (2020) 47-63.

[76] S. Zeki, 'Art and the brain', Daedalus 127 no. 2 (1998) 71-103. Reprinted in J. Consciousness Studies 6 (1999) 76-96. 